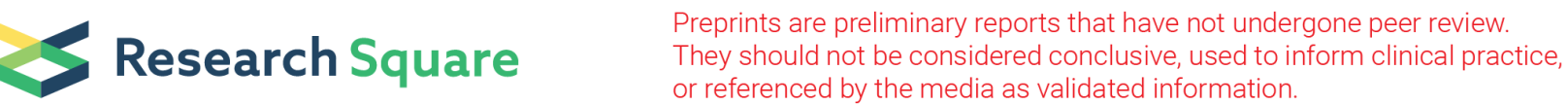

\section{Prognostic Value of Immune-Related genes in the Tumor Microenvironment of Glioma - Analyzed by Integrated Bioinformatics}

\section{Yali Zhong}

Guizhou University Of Traditional Chinese Medicine https://orcid.org/0000-0001-6223-4716

Xiaobin Luo

The Affiliated Hospital of Southwest Medical University

Fubing Yang

The Affiliated Hospital of Southwest Medical University

Xinling Song ( $20180299120312 @ s t u . s w m u . e d u . c n$ )

Guizhou University Of Traditional Chinese Medicine https://orcid.org/0000-0003-0690-6623

\section{Primary research}

Keywords: Glioma, Immune-Related Genes, Tumor Microenvironment, Stromal Score, Immune Score

Posted Date: February 18th, 2021

DOI: https://doi.org/10.21203/rs.3.rs-205389/v1

License: (c) (i) This work is licensed under a Creative Commons Attribution 4.0 International License. Read Full License 


\section{Abstract \\ Object:}

Immune related genes play an important role in the process of tumor genesis and development.

Therefore, we aim to find the Immune genes which are related to the prognosis of glioma patients, and to explore the infiltration of Immune cells in glioma microenvironment.

\section{Methods}

We downloaded the data of the glioma samples from the CGGA database, and performed batch correction to screen the primary glioma samples for subsequent analysis. Then the ESTIMATE algorithm was used to deal with the Stromal scores and Immune scores of the primary glioma samples, and the difference was analyzed. Then the common Immune related genes (IRGs) were obtained by intersecting with the Immune genes in the ImmPort database. Moreover, we used common IRGs to construct proteinprotein interaction (PPI) networks, from which we screened the top 30 genes with high connectivity, and Lasso regression was used to screen the IRGs. Lastly, we obtained the combined genes, which were overlapped both in the top 30 high-connection genes and Lasso regression genes. The final genes were used to construct COX risk prediction models. The accuracy of the model were verified by the TCGA glioma data, and the model genes were analyzed for Immune-related pathways, as well as the Hallmark and KEGG enrichment. Additionally, we used CIBERSOFT algorithm to estimate the Immune cell content of the samples, and analyzed the differences, correlations and survival of the Immune cells in high and low risk groups.

\section{Results}

Firstly, a total of 117 IRGs were obtained from the gene sets, which were overlapped in the data of Stromal score, Immune score and ImmPort database. Secondly, the top 30 genes were selected after the PPI network, and another 26 genes were screened out after the Lasso regression algorithm. And then, six coexist IRGs were obtained from the intersecting sets. Furthermore, the COX risk prediction model was constructed and tested, showing that the overall survival rate of the high-risk group was about $50 \%$ of that of the low-risk group. We observed that the high-risk group were enriched in Immune response and Immune process. Most importantly, in KEGG pathways, the high-risk groups were mainly enriched in p53 signaling pathway, JAK-STAT signaling pathway, pathways in cancer and cell cycle. By estimating the Immune cell contents, we also found that the Immune cell Plasma cells, T cells CD8, T cells CD4 naïve, T cells regulatory (Tregs), Macrophages M0 and Neutrophils were higher in high-risk groups, when compared to the low-risk group, with significant difference. Finally, the correlation analysis showed that the degree of Immune infiltration in high-risk groups was related to T cells regulatory (Tregs), Macrophages $\mathrm{M} 0$ and Neutrophils. 


\section{Conclusion}

A COX risk prediction model of 6 genes was successfully constructed, which was enriched in Immunerelated pathways. Meanwhile, survival analysis and TCGA data validation revealed significant differences in the model genes in the overall survival of the glioma patients, and the degree of Immune infiltration in the model was associated with T cells regulatory (Tregs), Macrophages M0 and Neutrophils.

\section{Introduction}

The glioblastoma is the most common primary malignant brain tumors, which is typically associated with a dismal prognosis and poor quality of life [1]. By 2019, the CBTRUS statistical reports found that the incidence of the central nervous system (CNS) tumors was about 23.41 per 100,000, and the mortality rate was about 442 per 100,000 [2]. At present, the glioma can be divided into two main types: diffuse glioma, which was the most common one, and non-diffusive glioma. World Health Organization (WHO) divided this malignancy into four grades, for grade $\otimes$ and grade $\otimes$ were the Low Grade Glioma (LGG), and grade $\nabla$ and grade $\otimes$ were the High Grade Glioma (HGG). Meanwhile, the grade $\otimes$ had the highest malignancy and extremely poor prognosis [3]. The systematic management for the tumor were including surgery, chemoradiotherapy, molecular targeting, Tumour Treating Fields (TTFs) therapy and so on [4].

For decades, immunotherapy has achieved fruitful results in the treatment of solid tumors, while is no remarkable breakthrough in the treatment of glioma, one of the might be the lack of the exploration and understanding of the effective Immune related genes (IRGs) and the Immune infiltration of glioma [5]. Recent studies have found that the growth and invasion of glioma are closely related to the Immune process and Immune response [6], while the underlying mechanism is still remain unclear.

The exploration of IRGs which were differentially expressed in the glioma patients with different prognosis can help us understand the pathogenesis of glioma, providing new ideas for the treatment of this malignancy and the management of the patients with this disease. In addition, tumor development was largely determined by the tumor microenvironment, which was an 'ecosystem' composed of Stromal cells, Immune cells, tumor stem cells and other appendages [6]. In previous studies, most of the studies mainly focused on the expression of tumor cell genes, but ignored the effects of the cell surrounding environment. However, it is found that Immune cells can promote and inhibit the invasion and growth of glioma. In brain glioma, microglia and macrophages can promote the growth, invasion and metastasis of glioma, which are associated with poor prognosis $[7,8]$, although the relationship between the Immune cells with the genes remains to be explored.

Thus, for the first time, we studied the relationship between IRGs and Immune cells, to reveal the role of immunity in glioma. At the same time, we also found the targeted polyenes, which would be the Immune prognostic markers of glioma. This study mainly used ESTIMATE algorithms to evaluate Stromal cells and Immune cells [9], and CIBERSOFT algorithms to estimate the infiltrating Immune cells in tumor samples [10]. Both of the two algorithms were successfully applied in the studies of urinary bladder 
carcinoma [11], lung cancer [12] and renal carcinoma [13], for which the reliability and accuracy were recognized.

In the present study, we downloaded primary glioma samples from the Chinese Glioma Genome Atlas (CGGA, http://www.cgga.org.cn/) database, and used the ESTIMATE algorithms to estimate Stromal cell and Immune cell scores in glioma sample, extracting the differentiated genes related to the scores. Then the co-expressed IRGs were obtained from the intersected gene sets with ImmPort Immune database. Multiple bioinformatics analyses were perform, and COX risk prediction model was constructed. Survival analysis, performance prediction and validation of the model were done, and the results indicated that the prognosis of the patients in high-risk group and the low-risk group of the prognostic model was significantly different. Moreover, pathway analysis showed that the targeted genes in high-risk groups were significantly enriched in Immune response and Immune related processes, and were associated with multiple Immune-related pathways in tumors. Finally, we analyzed the Immune microenvironment of glioma by CIBERSOFT algorithm, and found that in numerous kind of Immune cells, there were significant differences between the model of high-risk group and low-risk group. The article research process is shown in Fig. 1.

\section{Materials And Methods}

\subsection{Data collection and Processing}

At first, to explore the IRGs and Immune infiltration of tumor cells in the glioma Immune microenvironment, we collected mRNA-seq data from the CGGA database. The data were divided into two batches. Secondly, We combined the data for batch correction and selected the primary glioma samples for subsequent analysis. Thirdly, we used the ESTIMATE package for Stromal and Immune scoring of primary glioma samples. Finally, to compare the effects of the two scores on survival analysis, we took the median of the Stromal scores and divided the samples into the high Stromal score group and the low Stromal score group. The same method was used for the Immune scores and estimate scores. To analyze the correlations between clinical information and scores, we compared the clinical information correlations with Stromal scores, Immune scores, and estimate scores.

\subsection{Screening of co-differentially expressed IRGs and enrichment analysis}

Using the grouping principle in the previous survival analysis, the samples were divided into high Stromal score groups and low Stromal score groups according to the Stromal score's median value. Then the 'Limma' package was used for difference analyses. The final result was screened using the criteria of $|\mathrm{LogFC}| \geq 1$ and FDR $<0.001$ [14]. For differentially expressed Immune genes with Immune scores, the same methods and criteria were used for screening. In addition, to obtain the Immune genes, we screened the Immune genes through gene list (https://www.immport.org/shared/home) in the ImmPort database. After the duplicate Immune genes were deleted, they were crossed with the differential genes in the 
Stromal scores and Immune scores to obtain the common differential IRGs. To explore the role of codifferentially expressed IRGs in tumor immunity, we obtained the co-differentially expressed IRGs via the Venn diagram and used the 'org.hs.eg.db' package [15] to convert genelD into entrezID. The genes extracted from entrezID were analyzed for GO and KEGG pathways analysis using the 'cluster profiler' package, and the results were screened with a $p$-value $<0.05$.

\subsection{Construction of protein-protein interaction (PPI) network and risk prediction model}

The PPI network was built using the online web tool STRING (Version 11.0, https://string$\mathrm{db}$.org/cgi/input?sessionld=BBPrHBKJPUey\&input_page_show_search=off), setting the minimum required interaction score value to 0.4 and hide disconnected nodes in the network. Finally, the PPI network was imported into Cytoscape software (Version 3.8.0, https://cytoscape.org/). Its plug-in Cytohubba was used to screen for gene connectivity, which proved that the higher the degree of the connectivity was, the more critical the gene was in the network.

To construct the risk prediction model, we extract the expression level of the common differentially expressed IRGs and combine the expression level with each sample's survival time. Then the 'Survival' package [16], 'Survivminer' package [17], and 'Glmnet' package [18] was used for univariate Cox and Lasso regression analysis. The final result was intersected with the top 30 genes with the highest degree of connectivity in the PPI network. Finally, the common IRGs (target genes) of the two sets were used to construct a Cox risk prediction model, and the formula for calculating the risk prediction model was: Risk score $=\operatorname{Exp} 1 * \beta 1+\operatorname{Exp} 2 * \beta 2+\cdots \operatorname{Expn} * \beta n$. The risk values of each sample obtained by the above formula were divided into high-risk groups and low-risk groups by the median of the risk values, and then subjected to survival analysis and model prediction accuracy analysis. To verify the constructed model's accuracy, we downloaded 672 glioma samples from the TCGA database and screened 569 samples with complete survival data (Table 1). In the same way, the risk prediction model was constructed, and its predictive value and the model's accuracy were verified. 
Table 1

Comparison of clinical characteristics between training set and verification set

\begin{tabular}{|c|c|c|}
\hline & CGGA(Training set) & TCGA(Validation set) \\
\hline \multirow[t]{2}{*}{ Grade } & LGG(220, 35.14\%) & LGG(417, 73.29\%) \\
\hline & $\operatorname{HGG}(406,64.86 \%)$ & HGG(152, 26.71\%) \\
\hline \multirow[t]{2}{*}{ Gender } & Male(369,58.95\%) & Male(331,58.17\%) \\
\hline & Female(257,41.05\%) & Female(238,41.83\%) \\
\hline \multirow[t]{3}{*}{ Age } & $\leq 40(256,40.89 \%)$ & $\leq 40(236,41.48)$ \\
\hline & $>40(369,58.95 \%)$ & $>40(333,58.52)$ \\
\hline & unknow $(1,0.16 \%)$ & \\
\hline \multirow[t]{3}{*}{ Radio_status } & Treated(501,80.03\%) & \\
\hline & Un-treated(110,17.57\%) & \\
\hline & unknow(15,2.40\%) & \\
\hline \multirow[t]{3}{*}{ Chemo_status } & Treated(406,64.86\%) & \\
\hline & Un-treated(198,31.63\%) & \\
\hline & unknow(22,3.51\%) & \\
\hline \multirow[t]{3}{*}{ IDH_mutation_status } & Mutant(309,49.36\%) & \\
\hline & Wildtype(279,44.57\%) & \\
\hline & unknow(38,6.07\%) & \\
\hline \multirow[t]{3}{*}{ 1p19q_codeletion_status } & Codel(135,21.57\%) & \\
\hline & Non-codel(431,68.85\%) & \\
\hline & unknow(60,9.58\%) & \\
\hline \multirow[t]{3}{*}{ MGMTp_methylation_status } & methylated(286,45.69\%) & \\
\hline & un-methylated $(255,40.73 \%)$ & \\
\hline & unknow(85,13.58\%) & \\
\hline
\end{tabular}

\subsection{Gene set enrichment analyses (GSEA) for the model}

Through the above screening, we finally obtained six IRGs (CXCL10, IL6, MMP9, CCL5, CSF3, and IL2RA). To further understand the relationship between the risk prediction model's high and low-risk groups, and the related Immune pathways, we conducted pathway analysis on Immune Response (M19817) and Immune System Process (M13664). All analysis were based on Molecular Signaling Database v7.1 
(https://www.gsea-msigdb.org/gsea/msigdb/index.jsp). Similarly, we explored the Hallmark and KEGG pathways in gliomas and screened the analysis result with FDR q-val $<0.05$.

\subsection{Immune microenvironment of glioma}

To evaluate the Immune infiltration of glioma samples' Immune microenvironment, we analyzed the Immune microenvironment of all primary glioma samples using the online network tool CIBERSORT (https://cibersort.stanford.edu/). CIBERSORT is a commonly used tool for assessing the level of Immune cells in tumor samples, and it can assess the cell content of 22 Immune cells. Firstly, to further understand the correlation between Immune cells in the Immune microenvironment, we analyzed Immune cells' correlation in tumor samples. Secondly, to explore the difference in the relative content of Immune cells between the high-risk groups and low-risk groups, the 'Vioplot' package was used to analyze the difference between the high and low-risk groups. Thirdly, Immune cells related to Immune genes and risk scores were screened by correlation test. In addition, we performed survival analysis on all Immune cells in the Immune microenvironment. Finally, we used FunRich (version3.1.3, http://www.funrich.org/) to intersect the results of different analysis, correlation analysis, and survival analysis of Immune cells, and the Immune cells with different expressions, high correlation and impacts on the prognosis of patients with glioma were obtained .

\section{Result}

\subsection{Data processing}

The mRNA-seq data in the CGGA database were divided into two batches. A total of 1018 glioma samples were collected (including 426 cases of primary low-grade glioma, 199 cases of recurrent lowgrade glioma, 225 cases of primary glioblastoma, 133 cases of recurrent glioblastoma, 30 cases of secondary recurrent glioma, and 5 cases of incomplete information). To study the Immune microenvironment of primary gliomas, 626 patients with primary gliomas (including low-grade gliomas and high-grade gliomas) were screened for subsequent analysis. The survival analysis results of the Stromal score and the Immune score were both statistically different $(P<0.05)$ (Fig. 2). Meanwhile, the Stromal score, the Immune score and the composite score were correlated with the patient's Age, glioma's Grade, Radio status, Chemo status, MGMT P Methodology Status, IDH mutation status, and 1p19q codeletion status $(P<0.05)$ (Fig. 3\&4\&5).

\subsection{Screening common differentially expressed genes (DEGs) and enrichment analysis}

A total of 903 DEGs (including 684 up-regulated genes and 219 down-regulated genes) were obtained through difference analysis of Stromal scores. Similarly, 1006 DEGs (including 619 up-regulated genes and 387 down-regulated genes) were obtained through difference analysis of Immune scores. In the gene list of the ImmPort database, the duplicate genes were deleted, then the $1793 \mathrm{Immune}$ genes were obtained. Then 117 common IRGs (111 up-regulated genes and 6 down-regulated genes) (Fig. 6) 
(Table 2) were obtained form the intersection sets of the three sets. Evidently, the results of GO enrichment analysis (Fig. 7A) showed that IRGs in the biological process (BP) pathway was mainly enriched in humoral Immune response, cellular immunity, and lymphocyte-mediated immunity. Furthermore, the cellular component (CC) pathway was mainly enriched in immunoglobulin complex, blood microparticle, and cytoplasmic vesicle lumen. In molecular function (MF) pathway, it was mainly enriched in antigen binding, immunoglobulin receptor binding, and cytokine receptor binding. In addition, the results of the KEGG pathway enrichment analysis were also meaningfully, for the genes were mainly enriched in the Chemokine signaling pathway, TNF signaling pathway, and Antigen Processing and presentation (Fig. 7C). 
Table 2

Common IRGs

\begin{tabular}{|c|c|c|c|}
\hline EntrezID & Gene symbol & LogFC & Type \\
\hline 356 & FASLG & 1.4500398 & UP \\
\hline 366 & AQP9 & 1.4019409 & UP \\
\hline 728 & C5AR1 & 1.0545353 & UP \\
\hline 911 & CD1C & 1.616718 & UP \\
\hline 913 & CD1E & 1.207707 & UP \\
\hline 915 & CD3D & 1.469274 & UP \\
\hline 916 & CD3E & 1.5745611 & UP \\
\hline 917 & CD3G & 1.4094225 & UP \\
\hline 926 & CD8B & 1.4475447 & UP \\
\hline 940 & CD28 & 1.2412739 & UP \\
\hline 944 & TNFSF8 & 1.1334713 & UP \\
\hline 959 & CD40LG & 1.309975 & UP \\
\hline 962 & CD48 & 1.3072731 & UP \\
\hline 970 & CD70 & 1.7485801 & UP \\
\hline 1233 & CCR4 & 1.2667997 & UP \\
\hline 1234 & CCR5 & 1.6095804 & UP \\
\hline 1236 & CCR7 & 1.3245825 & UP \\
\hline 1439 & CSF2RB & 1.1343373 & UP \\
\hline 1440 & CSF3 & 1.4229783 & UP \\
\hline 2069 & EREG & 1.582563 & UP \\
\hline 2213 & FCGR2B & 1.7418714 & UP \\
\hline 2358 & FPR2 & 1.510351 & UP \\
\hline 2740 & GLP1R & -1.275372 & DOWN \\
\hline 2833 & CXCR3 & 2.0329117 & UP \\
\hline 2834 & PRLHR & -1.324264 & DOWN \\
\hline 2919 & CXCL1 & 1.0866993 & UP \\
\hline 3002 & GZMB & 1.0780927 & UP \\
\hline
\end{tabular}




\begin{tabular}{|c|c|c|c|}
\hline EntrezID & Gene symbol & LogFC & Type \\
\hline 3111 & HLA-DOA & 1.0875023 & UP \\
\hline 3117 & HLA-DQA1 & 1.0520745 & UP \\
\hline 3118 & HLA-DQA2 & 1.7437905 & UP \\
\hline 3310 & HSPA6 & 1.277651 & UP \\
\hline 3350 & HTR1A & -1.435082 & DOWN \\
\hline 3493 & IGHA1 & 1.8441607 & UP \\
\hline 3494 & IGHA2 & 2.4552253 & UP \\
\hline 3495 & IGHD & 1.8525539 & UP \\
\hline 3500 & IGHG1 & 1.675743 & UP \\
\hline 3501 & IGHG2 & 2.2002078 & UP \\
\hline 3502 & IGHG3 & 2.1127739 & UP \\
\hline 3503 & IGHG4 & 2.4388214 & UP \\
\hline 3507 & IGHM & 2.0109422 & UP \\
\hline 3514 & IGKC & 1.2025322 & UP \\
\hline 3537 & IGLC1 & 1.960524 & UP \\
\hline 3538 & IGLC2 & 2.1291725 & UP \\
\hline 3539 & IGLC3 & 1.8887392 & UP \\
\hline 3552 & IL1A & 1.1117646 & UP \\
\hline 3557 & IL1RN & 1.7661992 & UP \\
\hline 3559 & IL2RA & 2.418335 & UP \\
\hline 3560 & IL2RB & 1.4683773 & UP \\
\hline 3569 & IL6 & 1.6431188 & UP \\
\hline 3575 & IL7R & 1.3642022 & UP \\
\hline 3586 & IL10 & 1.5801653 & UP \\
\hline 3620 & ID01 & 2.233656 & UP \\
\hline 3627 & CXCL10 & 1.5886023 & UP \\
\hline 3702 & ITK & 1.3922469 & UP \\
\hline 3929 & LBP & 2.2292246 & UP \\
\hline
\end{tabular}




\begin{tabular}{|c|c|c|c|}
\hline EntrezID & Gene symbol & LogFC & Type \\
\hline 3932 & LCK & 1.1985862 & UP \\
\hline 3976 & LIF & 1.7453125 & UP \\
\hline 4057 & LTF & 1.8420353 & UP \\
\hline 4068 & SH2D1A & 1.4898744 & UP \\
\hline 4069 & LYZ & 1.073843 & UP \\
\hline 4261 & CIITA & 1.0250743 & UP \\
\hline 4283 & CXCL9 & 1.8818678 & UP \\
\hline 4318 & MMP9 & 1.7189808 & UP \\
\hline 4481 & MSR1 & 1.0833277 & UP \\
\hline 4982 & TNFRSF11B & 1.2540348 & UP \\
\hline 5008 & OSM & 1.3063417 & UP \\
\hline 5133 & PDCD1 & 1.6241525 & UP \\
\hline 5266 & $\mathrm{PI} 3$ & 2.2685738 & UP \\
\hline 5320 & PLA2G2A & 2.467812 & UP \\
\hline 5328 & PLAU & 1.2126974 & UP \\
\hline 5551 & PRF1 & 1.4324096 & UP \\
\hline 5732 & PTGER2 & 1.1701595 & UP \\
\hline 5734 & PTGER4 & 1.2576062 & UP \\
\hline 5806 & PTX3 & 1.3158935 & UP \\
\hline 6036 & RNASE2 & 1.468534 & UP \\
\hline 6037 & RNASE3 & 1.3310565 & UP \\
\hline 6288 & SAA1 & 2.3840865 & UP \\
\hline 6289 & SAA2 & 2.7055376 & UP \\
\hline 6352 & CCL5 & 1.1358001 & UP \\
\hline 6355 & CCL8 & 1.3746357 & UP \\
\hline 6362 & CCL18 & 1.7075112 & UP \\
\hline 6364 & CCL20 & 2.0049368 & UP \\
\hline 6372 & CXCL6 & 2.0293719 & UP \\
\hline
\end{tabular}




\begin{tabular}{|c|c|c|c|}
\hline EntrezID & Gene symbol & LogFC & Type \\
\hline 6373 & CXCL11 & 1.2046347 & UP \\
\hline 6375 & XCL1 & 1.7990128 & UP \\
\hline 6398 & SECTM1 & 1.0139096 & UP \\
\hline 6751 & SSTR1 & -1.059583 & DOWN \\
\hline 6781 & STC1 & 1.8104046 & UP \\
\hline 6846 & XCL2 & 2.0725446 & UP \\
\hline 7044 & LEFTY2 & 1.216262 & UP \\
\hline 7421 & VDR & 1.5310255 & UP \\
\hline 7850 & IL1R2 & 1.1458052 & UP \\
\hline 8638 & OASL & 1.111828 & UP \\
\hline 8685 & MARCO & 1.116388 & UP \\
\hline 8740 & TNFSF14 & 2.1508323 & UP \\
\hline 8807 & IL18RAP & 1.1145595 & UP \\
\hline 9021 & socs3 & 1.2373531 & UP \\
\hline 9518 & GDF15 & 1.3047516 & UP \\
\hline 9966 & TNFSF15 & 1.2259036 & UP \\
\hline 10344 & CCL26 & 1.3053515 & UP \\
\hline 10663 & CXCR6 & 1.6236838 & UP \\
\hline 10911 & UTS2 & 1.6816861 & UP \\
\hline 11025 & LILRB3 & 1.4582705 & UP \\
\hline 11082 & ESM1 & 1.5301283 & UP \\
\hline 22943 & DKK1 & 1.114845 & UP \\
\hline 23529 & CLCF1 & 1.6025716 & UP \\
\hline 28526 & TRDC & 1.1819897 & UP \\
\hline 28755 & TRAC & 1.3772939 & UP \\
\hline 28820 & IGLV1-51 & 1.561688 & UP \\
\hline 51311 & TLR8 & 1.5785487 & UP \\
\hline 56729 & RETN & 1.8425855 & UP \\
\hline
\end{tabular}




\begin{tabular}{|llll|}
\hline EntrezID & Gene symbol & LogFC & Type \\
\hline 59350 & RXFP1 & -1.288158 & DOWN \\
\hline 84539 & MCHR2 & -1.761674 & DOWN \\
90226 & UCN2 & 1.2828467 & UP \\
\hline 131177 & FAM3D & 1.4067151 & UP \\
149954 & BPIFB4 & 1.6968206 & UP \\
164668 & APOBEC3H & 1.2303031 & UP \\
\hline
\end{tabular}

\subsection{Building PPI network and risk prediction model}

Using the online web tool STRING, we constructed the PPI network of 117 IRGs, and obtained a PPI network of 97 nodes and 873 edges. The top 30 genes with high connectivity were selected by screening the network's gene connectivity (Fig. 8C). The Lasso analysis of the 117 IRGs resulted in 26 genes (Fig. 8A\&B). Then six IRGs (CXCL10, IL6, MMP9, CCL5, CSF3, and IL2Ra) were obtained form the intersection of the two sets (Fig. 8D). Subsequently, we used the six IRGs to construct a Cox risk prediction model and analyze the model's prediction accuracy (Fig. 8E). The results showed that there was a significant difference in prognosis between the high-risk groups and the low-risk groups $(P<0.001)$, and the ROC curve showed that the prediction accuracy of the model within five years was greater than 0.8 (area under curve,AUC) (Fig. 9A\&B). Finally, sample validation using TCGA glioma revealed a significant difference in prognosis between the high and low-risk groups $(P<0.001)$, with an AUC under the ROC curve of $>0.8$, suggesting that the model was highly accurate in prediction (Fig. 9C\&D).

\subsection{Functional enrichment analysis of the model}

The results of the pathway analyze showed that the high-risk group was more likely to accumulate on the Immune Response and Immune System Process pathways than the low-risk group, which indicated that the higher the risk score was, the more intense the Immune Response process was (Fig. 10A\&B). Analysis of the glioma pathway at Hallmark showed that the high-risk group genes were mainly enriched in Inflammatory Response, p53 Pathway, and PI3k Akt Mtor Signaling (Fig. 10C). In the KEGG pathway analyze, the high-risk group genes were more likely enriched in p53 signaling pathway, JAK-STAT signaling pathway, antigen processing and presentation, pathways in cancer, and cell cycle (Fig. 10D).

\subsection{Immune microenvironment analysis}

Using the online network tool of CIBERSORT, we estimated the relative content of 22 Immune cells in 626 glioma samples (Fig. 11). The results showed that the relative content of Macrophages M0, Macrophages M2, Mast cells resting, and Mast cells activated was more than 0.45 . The results of the difference analysis showed that the contents of T cells follicular helper, NK cells resting, and Monocytes in the lowrisk group were higher than those in the high-risk group (Fig. 11B). Furthermore, the high-risk group had higher level of Plasma cells, T cells CD8, T cells CD4 naïve, T cells regulatory (Tregs), Macrophages M0, 
Macrophages M1, and Neutrophils than the low-risk group (Fig. 11B). The correlation between the risk score of the predictive model and Immune cells was also analyzed. The results showed that the risk score had a positive correlation with T cells regulatory (Tregs), Eosinophils, Macrophages M1, Neutrophils, T cells follicular helper and T cells gamma delta, and the positive correlation with Macrophages M0 was the highest $(R=0.72, p<2.2 e-16)$. In contrast, it was negatively correlated with $T$ cells CD 4 memory recall and T cells CD4 naive, and was most negatively correlated with Moncyte $(R=-0.6, p<2.2 e-16)$. Finally, we obtained five types of Immune cells (Fig. 12) through the intersected sets of the difference analysis, correlation analysis, and survival analysis, wherein the Macrophages M0, Neutrophils, and T cells regulatory (Tregs) cells were significantly correlated with the prognosis of glioma (Fig. 13F\&g\&H). Interestingly, the higher the relative amounts of Monocytes and T cells CD4 naïve (Fig. 13I\&J), the higher the survival rate of patients with gliomas.

\section{Discussion}

Glioma is a primary tumor of the brain, which accounting for about $81 \%$ of intracranial malignancies. According to the invasiveness of the tumor, WHO divided the glioma into I-IV grades, while the grade IV glioma had the highest degree of malignancy, of which the five-year survival rate was about 5 percent and the median survival time was about $12-15$ months $[19,20]$. Because the tumor microenvironment of glioma survival is too complex, it brings challenges to our research and treatment, which leads to poor clinical therapeutic effect and even therapeutic resistance [21]. In recent years, with the development of bioinformatics and the multi-omics researches of tumor, we have a deeper understanding of the process of tumorigenesis and development. Immune infiltration plays an important role in the development of glioma cells, which recruits immune cells for their growth by secreting chemokines [22], although the specific roles of immune cells in glioma still remains unclear. In line with the recent success of immunotherapy in multiple solid tumor [23], it is meaningful to explore the immune related genes of glioma and clarify the infiltration of immune cells in tumors.

CGGA database is constructed for offering convenience for the researchers to exploring the pathological mechanisms, molecular typing and therapeutic targets for glioma. Meanwhile, ImmPort database collected comprehensive immunological data [24]. By downloading the data of glioma samples from the CGGA database, and performing preliminary screen, we obtained the primary glioma samples. The score of the stromal cells and immune cells in the glioma samples was calculated by ESTIMATE algorithm. Then the 117 IRGs were obtained from the intersected sets with the Immune related genes in the ImmPort database. GO enrichment analysis showed that the target genes enrich in many important immunerelated pathways. For examples, BP pathway is mainly enriched in humoral immune response, cellular immunity and lymphocyte mediated immunity. MF pathway is mainly enriched in antigen binding, immunoglobulin receptor binding and cytokine receptor binding, which suggested IRGs in gliomas are involved in the Immune response, and might be associated with glioma progression and growth. The KEGG pathway analysis indicated the IRGs were mainly enriched in JAK-STAT signaling pathway, IL - 17 signaling pathway, Toll - like receptor signaling pathway and so on. Interestingly, JAK-STAT signaling pathway is closely related to cell growth and differentiation. Meanwhile, the STAT protein family have a 
variety of proteins, as STAT3 and STAT5, which are closely related to tumor immune response, tumor invasion and tumor spreading $[25,26]$. Xu et al. found that silencing microrna-221/222 clusters could inhibit JAK/STAT3 pathways, then inhibiting GBM angiogenesis [27]. Zhang et al. proved that the upregulation of mir-133a could inhibit expression of CTGF and the activation of the signaling pathway, and consequently, the glioma proliferation, migration and invasion were inhibited [28]. IL-17, a highly versatile pro-inflammatory cytokine, is crucial for a variety of pathophysiological processes, including host defense, tissue repair, the pathogenesis of inflammatory disease, as well as the progression of cancer. In the short and medium term, IL-17 could promote tissue repair and defense, while aggravate the cancer development and autoimmunity in long-term [29]. Wang et al. examined that by activating of PI3K/Akt1/NF-KB-p65 pathways, IL-17 could induce glioma cell proliferation and migration [30]. Moreover, You et al. found that the expression of astrocytes and vascular endothelial growth factor (VEGF) could be induced by IL-17 through JAK/STAT pathway in patients with spinal cord injury [31]. Toll-like receptors (TLRs) performs significant roles in controlling Immune functions, for the function, regulation and pattern expression. Signals produced by Toll-like receptors could be transduced through NF-KB and MAP kinase pathways to aggravate inflammation and tumorigenesis [32]. In line with this, Li et al, and other studies have found that LnCRNA CRNDE may trigger inflammation through Toll-like receptor pathway to regulate tumorigenesis and tumor development [33]. To sum up, the above evidences suggested that the IRGs could promote glioma proliferation, migration and invasion through multiple pathways.

By constructing the risk prediction model, we obtained 6 mRNA(CXCL10, IL6, MMP9, CCL5, CSF3, IL2RA). Evidence revealed that the CXCL10 genes were overexpressed in glioblastoma, and were associated with its growth and progression [34]. Maru et al. revealed that CXCL10 could induce the Erk1/2 increase and uptake of $\left[{ }^{3} \mathrm{H}\right]$ thymidine, then promoting glioma cell proliferation [35]. The level of IL-6 mRNA was reported to have relationship with prognosis and survival of the glioma patients [36]. What's more, through the JAK/STAT3 pathway, tumor growth may be promoted [37]. Meanwhile, it was also found that IL-6 can also promote the expression of MMP9 in U251 and T98G cell lines [37]. Recent studies have proved that IL- 6 can induce the production of $\mathrm{T}_{H} 17$ cells and myeloid-derived suppressor cells (MDSCs) to promote tumorigenesis, as well as transforming the tumor-associated tumorigenic M1 macrophages into the immunosuppression-related M2 macrophages [38]. The hypoxia and inflammation stimulated the overexpression of MMP9, which is closely related to the glioma progression $[39,40]$. CCL5/CCR5 axis was reported to promote glioma cell proliferation activates through PI3K/Akt pathway [41, 42]. Recently, researchers found that CCL5 expression is associated with poor prognosis in LGG patients [43]. Gao et al. found that CSF3 gene is involved in inflammatory reactions and is the GBM diagnostic plasma markers [44]. More importantly, IL2RA gene receptors are reported to locate on the surface of regulatory T cells and may be involved in immunosuppression of GBM tumor microenvironment through cytokine signaling pathways [45]. Moreover, through GSEA analysis of the high and low risk groups in risk prediction models, we found that the high risk groups were mainly enriched in the Immune response related processes, which suggested that the Immune response was reacted intensively in glioma prognostic models. Meanwhile, in Hallmark and KEGG pathways are enriched p53 signaling pathway, which is mainly related to cell cycle and apoptosis [45]. In KEGG pathways, we also found the high-risk groups significantly 
enriched in JAK-STAT signaling pathway, antigen processing and presentation, pathways in cancer and cell cycle.

The tumor microenvironment (TME) refers to the cell environment in which tumor cells or tumor stem cells exist [46], which is mainly composed of Stromal cells, endothelial cells, Immune cells and other accessory cells [47]. Its main function is to participate in the tumor vascular survival, promoting invasion and metastasis [48]. In the microenvironment of glioma, tumor-associated macrophages (TAM) and microglia played key roles, while the inhibition of TAM could intervene the growth of glioma [49]. Moreover, TAM is also involved in the angiogenesis in brain tumors and the drug resistance of antiangiogenic drugs [50]. Prior to that, TAM were generally divided into M1 and M2 two phenotypes. However, Gabrusiewicz proposed that there were still a large number of non-polarized M0 macrophages in the GBM region, and found that CD14 + cells were very similar to M0 macrophages, which may be related to the Immune infiltration of GBM [51]. In addition, Haung et al. found that the higher levels of M0 macrophages in the glioma samples [52],which was consistent with our findings. They also suggested that the expression level of EFEMP2 was associated with M0 macrophage cell assembly, and that EFEMP2 could also act as a chemokine for TAM. Bertaut et al. found that the Neutrophils count in peripheral blood of GBM patients could predict the efficacy of bevacizumab [53]. Moreover, Liang et al. suggested that Neutrophils infiltration was associated with glioma progression and drug resistance during anti-VEGF therapy [54]. T cells Regulatory (Tregs) plays a crucial role in normal immunity, mediating autotolerance [55], and regulating the function of Immune cells. In the research of the cancer field, it is found that overactivity of the Tregs is associated with tumor cell escape [56]. Meanwhile, there is also a correlation between the degree of invasion of Tregs and WHO classification of the tumor [57]. In addition, Tregs recruitment is evident in glioma mouse models, suggesting that it might be necessary for tumor growth. Therefore, targeted anti-Treg therapy was proposed, and its effectiveness was verified in glioma mouse experiment and GBM patient treatment $[58,59]$. Monocytes can be transformed into tumorrelated macrophages to perform roles, while the relevant mechanism remains to uncover [60].

\section{Conclusion}

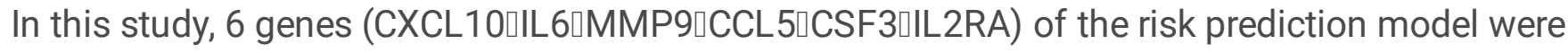
constructed by scoring Immune cells and Stromal cells, and the predictive ability of the model is verified. The GSEA analysis of the model showed that the high-risk group of the model was significantly enriched in Immune-related pathways. In addition, we also estimated the content of Immune cells in glioma, and focus on Macrophages M0, Neutrophils and T Cells Regulatory Tregs, of which the contents were negatively correlated with the prognosis of patients. Combined with relevant studies, we concluded that the higher contents of the three kinds of Immune cells were related to the stronger the Immune infiltration of glioma, consequently, the causing worse prognosis of the patients.

\section{Ethics approval and consent to participate}

Not applicable. 


\section{Consent for publication}

All authors agreed on the manuscript.

\section{Availability of data and materials}

Data used to support the results of this study can be obtained from the corresponding authors as required.

\section{Competing interests}

The authors declare that they have no competing interests.

\section{Funding}

Not applicable

\section{Authors' contributions}

Yali Zhong and Xiaobin Luo: Conceptualization, Methodology, Formal analysis, Validation, Writing review \& editing. Fubing Yang and Xinling Song: Supervision, Writing - review \& editing, Project administration.

\section{Acknowledgements}

We thank all team members of TCGA and CGGA projects for providing public-access data.

\section{Declarations}

\section{Ethics approval and consent to participate}

Not applicable.

\section{Consent for publication}

All authors agreed on the manuscript.

\section{Availability of data and materials}

Data used to support the results of this study can be obtained from the corresponding authors as required.

\section{Competing interests}

The authors declare that they have no competing interests.

\section{Funding}


Not applicable

\section{Authors' contributions}

Yali Zhong and Xiaobin Luo: Conceptualization, Methodology, Formal analysis, Validation, Writing review \& editing. Fubing Yang and Xinling Song: Supervision, Writing - review \& editing, Project administration.

\section{Acknowledgements}

We thank all team members of TCGA and CGGA projects for providing public-access data.

\section{References}

1. Wesseling P, Capper D. WHO 2016 Classification of gliomas. Neuropathol Appl Neurobiol. 2018;44:139-50.

2. Ostrom QT, Cioffi G, Gittleman H, Patil N, Waite K, Kruchko C, et al. CBTRUS Statistical Report: Primary Brain and Other Central Nervous System Tumors Diagnosed in the United States in 20122016. Neuro-oncology. 2019;21:v1-100.

3. Li K, Lu D, Guo Y, Wang C, Liu X, Liu Y, et al. Trends and patterns of incidence of diffuse glioma in adults in the United States, 1973-2014. Cancer Med. 2018;7:5281-90.

4. Batash R, Asna N, Schaffer P, Francis N, Schaffer M. Glioblastoma Multiforme, Diagnosis and Treatment; Recent Literature Review. Curr Med Chem. 2017;24:3002-9.

5. Fecci PE, Sampson JH. The current state of immunotherapy for gliomas: an eye toward the future. J Neurosurg. 2019;131:657-66.

6. Miyauchi JT, Tsirka SE. Advances in Immunotherapeutic Research for Glioma Therapy. J Neurol. 2018;265:741-56.

7. Carvalho da Fonseca AC, Badie B. Microglia and Macrophages in Malignant Gliomas: Recent Discoveries and Implications for Promising Therapies. Clin Dev Immunol [Internet]. 2013 [cited 2020 Sep 7];2013. Available from: https://www.ncbi.nlm.nih.gov/pmc/articles/PMC3707269/

8. Roesch S, Rapp C, Dettling S, Herold-Mende C. When Immune Cells Turn Bad-Tumor-Associated Microglia/Macrophages in Glioma. Int J Mol Sci [Internet]. 2018 [cited 2020 Sep 7];19. Available from: https://www.ncbi.nlm.nih.gov/pmc/articles/PMC5855658/

9. Yoshihara K, Shahmoradgoli M, Martínez E, Vegesna R, Kim H, Torres-Garcia W, et al. Inferring tumour purity and stromal and immune cell admixture from expression data. Nat Commun. 2013;4:2612.

10. Chen B, Khodadoust MS, Liu CL, Newman AM, Alizadeh AA. Profiling Tumor Infiltrating Immune Cells with CIBERSORT. Methods Mol Biol. 2018;1711:243-59.

11. Li F, Teng H, Liu M, Liu B, Zhang D, Xu Z, et al. Prognostic Value of Immune-Related Genes in the Tumor Microenvironment of Bladder Cancer. Front Oncol. 2020;10:1302. 
12. Mony JT, Schuchert MJ. Prognostic Implications of Heterogeneity in Intra-tumoral Immune Composition for Recurrence in Early Stage Lung Cancer. Front Immunol. 2018;9:2298.

13. Zhang S, Zhang E, Long J, Hu Z, Peng J, Liu L, et al. Immune infiltration in renal cell carcinoma. Cancer Sci. 2019;110:1564-72.

14. Ritchie ME, Phipson B, Wu D, Hu Y, Law CW, Shi W, et al. limma powers differential expression analyses for RNA-sequencing and microarray studies. Nucleic Acids Res. 2015;43:e47.

15. Carlson M. org.Hs.eg.db: Genome wide annotation for Human. 2020.

16. Therneau TM, Grambsch PM. Modeling Survival Data: Extending the Cox Model [Internet]. New York: Springer-Verlag; 2000 [cited 2020 Jul 22]. Available from: https://www.springer.com/it/book/9780387987842

17. Kassambara A, Kosinski M, Biecek P. survminer: Drawing Survival Curves using "ggplot2" [Internet]. 2020. Available from: https://CRAN.R-project.org/package=survminer

18. Friedman J, Hastie T, Tibshirani R. Regularization Paths for Generalized Linear Models via Coordinate Descent. J Stat Softw. 2010;33:1-22.

19. Ostrom QT, Bauchet L, Davis FG, Deltour I, Fisher JL, Langer CE, et al. The epidemiology of glioma in adults: a "state of the science" review. Neuro Oncol. 2014;16:896-913.

20. Soomro SH, Ting LR, Qing YY, Ren M. Molecular biology of glioblastoma: Classification and mutational locations. J Pak Med Assoc. 2017;67:1410-4.

21. Mackay A, Burford A, Carvalho D, Izquierdo E, Fazal-Salom J, Taylor KR, et al. Integrated Molecular Meta-Analysis of 1,000 Pediatric High-Grade and Diffuse Intrinsic Pontine Glioma. Cancer Cell. 2017;32:520-537.e5.

22. Gutmann DH. The Sociobiology of Brain Tumors. Adv Exp Med Biol. 2020;1225:115-25.

23. Majd N, Dasgupta P, de Groot J. Immunotherapy for Neuro-Oncology. Adv Exp Med Biol. 2020;1244:183-203.

24. Bhattacharya S, Dunn P, Thomas CG, Smith B, Schaefer H, Chen J, et al. ImmPort, toward repurposing of open access immunological assay data for translational and clinical research. Sci Data. 2018;5:180015.

25. Chang WH, Lai AG. An immunoevasive strategy through clinically-relevant pan-cancer genomic and transcriptomic alterations of JAK-STAT signaling components. Mol Med [Internet]. 2019 [cited 2020 Sep 9];25. Available from: https://www.ncbi.nlm.nih.gov/pmc/articles/PMC6829980/

26. Swiatek-Machado K, Kaminska B. STAT Signaling in Glioma Cells. Adv Exp Med Biol. 2020;1202:203-22.

27. Xu C-H, Liu Y, Xiao L-M, Chen L-K, Zheng S-Y, Zeng E-M, et al. Silencing microRNA-221/222 cluster suppresses glioblastoma angiogenesis by suppressor of cytokine signaling-3-dependent JAK/STAT pathway. J Cell Physiol. 2019;234:22272-84.

28. Zhang P, Chen F-Z, Jia Q-B, Hu D-F. Upregulation of microRNA-133a and downregulation of connective tissue growth factor suppress cell proliferation, migration, and invasion in human glioma 
through the JAK/STAT signaling pathway. IUBMB Life. 2019;71:1857-75.

29. Li X, Bechara R, Zhao J, McGeachy MJ, Gaffen SL. IL-17 receptor-based signaling and implications for disease. Nat Immunol. 2019;20:1594-602.

30. Wang B, Zhao C-H, Sun G, Zhang Z-W, Qian B-M, Zhu Y-F, et al. IL-17 induces the proliferation and migration of glioma cells through the activation of PI3K/Akt1/NF-KB-p65. Cancer Lett. 2019;447:93104.

31. You T, Bi Y, Li J, Zhang M, Chen X, Zhang K, et al. IL-17 induces reactive astrocytes and up-regulation of vascular endothelial growth factor (VEGF) through JAK/STAT signaling. Sci Rep. 2017;7:41779.

32. Vidya MK, Kumar VG, Sejian V, Bagath M, Krishnan G, Bhatta R. Toll-like receptors: Significance, ligands, signaling pathways, and functions in mammals. Int Rev Immunol. 2018;37:20-36.

33. Li H, Li Q, Guo T, He W, Dong C, Wang Y. LncRNA CRNDE triggers inflammation through the TLR3-NFKB-Cytokine signaling pathway. Tumour Biol. 2017;39:1010428317703821.

34. Sharma I, Siraj F, Sharma KC, Singh A. Immunohistochemical expression of chemokine receptor CXCR3 and its ligand CXCL10 in low-grade astrocytomas and glioblastoma multiforme: A tissue microarray-based comparison. J Cancer Res Ther. 2016;12:793-7.

35. Maru SV, Holloway KA, Flynn G, Lancashire CL, Loughlin AJ, Male DK, et al. Chemokine production and chemokine receptor expression by human glioma cells: role of CXCL10 in tumour cell proliferation. J Neuroimmunol. 2008;199:35-45.

36. Chang C-Y, Li M-C, Liao S-L, Huang Y-L, Shen C-C, Pan H-C. Prognostic and clinical implication of IL-6 expression in glioblastoma multiforme. J Clin Neurosci. 2005;12:930-3.

37. Liu Q, Li G, Li R, Shen J, He Q, Deng L, et al. IL-6 promotion of glioblastoma cell invasion and angiogenesis in U251 and T98G cell lines. J Neurooncol. 2010;100:165-76.

38. Jones SA, Jenkins BJ. Recent insights into targeting the IL-6 cytokine family in inflammatory diseases and cancer. Nat Rev Immunol. 2018;18:773-89.

39. Barillari G. The Impact of Matrix Metalloproteinase-9 on the Sequential Steps of the Metastatic Process. Int J Mol Sci. 2020;21.

40. Levicar N, Nuttall RK, Lah TT, Nutall RK. Proteases in brain tumour progression. Acta Neurochir (Wien). 2003;145:825-38.

41. Zhao L, Wang Y, Xue Y, Lv W, Zhang Y, He S. Critical roles of chemokine receptor CCR5 in regulating glioblastoma proliferation and invasion. Acta Biochim Biophys Sin (Shanghai). 2015;47:890-8.

42. Kranjc MK, Novak M, Pestell RG, Lah TT. Cytokine CCL5 and Receptor CCR5 Axis in Glioblastoma Multiforme. Radiol Oncol. 2019;53:397-406.

43. Guo X, Pan Y, Xiong M, Sanapala S, Anastasaki C, Cobb O, et al. Midkine activation of CD8+ T cells establishes a neuron-immune-cancer axis responsible for low-grade glioma growth. Nat Commun. 2020;11:2177.

44. Gao Y, Zhang E, Liu B, Zhou K, He S, Feng L, et al. Integrated analysis identified core signal pathways and hypoxic characteristics of human glioblastoma. J Cell Mol Med. 2019;23:6228-37. 
45. Schwartzbaum JA, Xiao Y, Liu Y, Tsavachidis S, Berger MS, Bondy ML, et al. Inherited variation in immune genes and pathways and glioblastoma risk. Carcinogenesis. 2010;31:1770-7.

46. Arneth B. Tumor Microenvironment. Medicina (Kaunas). 2019;56.

47. Kim J, Bae J-S. Tumor-Associated Macrophages and Neutrophils in Tumor Microenvironment. Mediators Inflamm [Internet]. 2016 [cited 2020 Sep 19];2016. Available from:

https://www.ncbi.nlm.nih.gov/pmc/articles/PMC4757693/

48. Denton AE, Roberts EW, Fearon DT. Stromal Cells in the Tumor Microenvironment. Adv Exp Med Biol. 2018;1060:99-114.

49. Pyonteck SM, Akkari L, Schuhmacher AJ, Bowman RL, Sevenich L, Quail DF, et al. CSF-1R inhibition alters macrophage polarization and blocks glioma progression. Nat Med. 2013;19:1264-72.

50. Lu-Emerson C, Snuderl M, Kirkpatrick ND, Goveia J, Davidson C, Huang Y, et al. Increase in tumorassociated macrophages after antiangiogenic therapy is associated with poor survival among patients with recurrent glioblastoma. Neuro-oncology. 2013;15:1079-87.

51. Gabrusiewicz K, Rodriguez B, Wei J, Hashimoto Y, Healy LM, Maiti SN, et al. Glioblastoma-infiltrated innate immune cells resemble MO macrophage phenotype. JCI Insight. 2016;1.

52. Huang L, Wang Z, Chang Y, Wang K, Kang X, Huang R, et al. EFEMP2 indicates assembly of M0 macrophage and more malignant phenotypes of glioma. Aging (Albany NY). 2020;12:8397-412.

53. Bertaut A, Truntzer C, Madkouri R, Kaderbhai CG, Derangère V, Vincent J, et al. Blood baseline neutrophil count predicts bevacizumab efficacy in glioblastoma. Oncotarget. 2016;7:70948-58.

54. Liang J, Piao Y, Holmes L, Fuller GN, Henry V, Tiao N, et al. Neutrophils promote the malignant glioma phenotype through S100A4. Clin Cancer Res. 2014;20:187-98.

55. Lowther DE, Hafler DA. Regulatory T cells in the central nervous system. Immunol Rev. 2012;248:156-69.

56. Wolf AM, Wolf D, Steurer M, Gastl G, Gunsilius E, Grubeck-Loebenstein B. Increase of regulatory T cells in the peripheral blood of cancer patients. Clin Cancer Res. 2003;9:606-12.

57. Jacobs JFM, Idema AJ, Bol KF, Grotenhuis JA, de Vries IJM, Wesseling P, et al. Prognostic significance and mechanism of Treg infiltration in human brain tumors. J Neuroimmunol. 2010;225:195-9.

58. Grauer OM, Nierkens S, Bennink E, Toonen LWJ, Boon L, Wesseling P, et al. CD4+FoxP3+ regulatory T cells gradually accumulate in gliomas during tumor growth and efficiently suppress antiglioma immune responses in vivo. Int J Cancer. 2007;121:95-105.

59. Fecci PE, Mitchell DA, Whitesides JF, Xie W, Friedman AH, Archer GE, et al. Increased regulatory T-cell fraction amidst a diminished CD4 compartment explains cellular immune defects in patients with malignant glioma. Cancer Res. 2006;66:3294-302.

60. Guilliams M, Mildner A, Yona S. Developmental and Functional Heterogeneity of Monocytes. Immunity. Elsevier; 2018;49:595-613. 


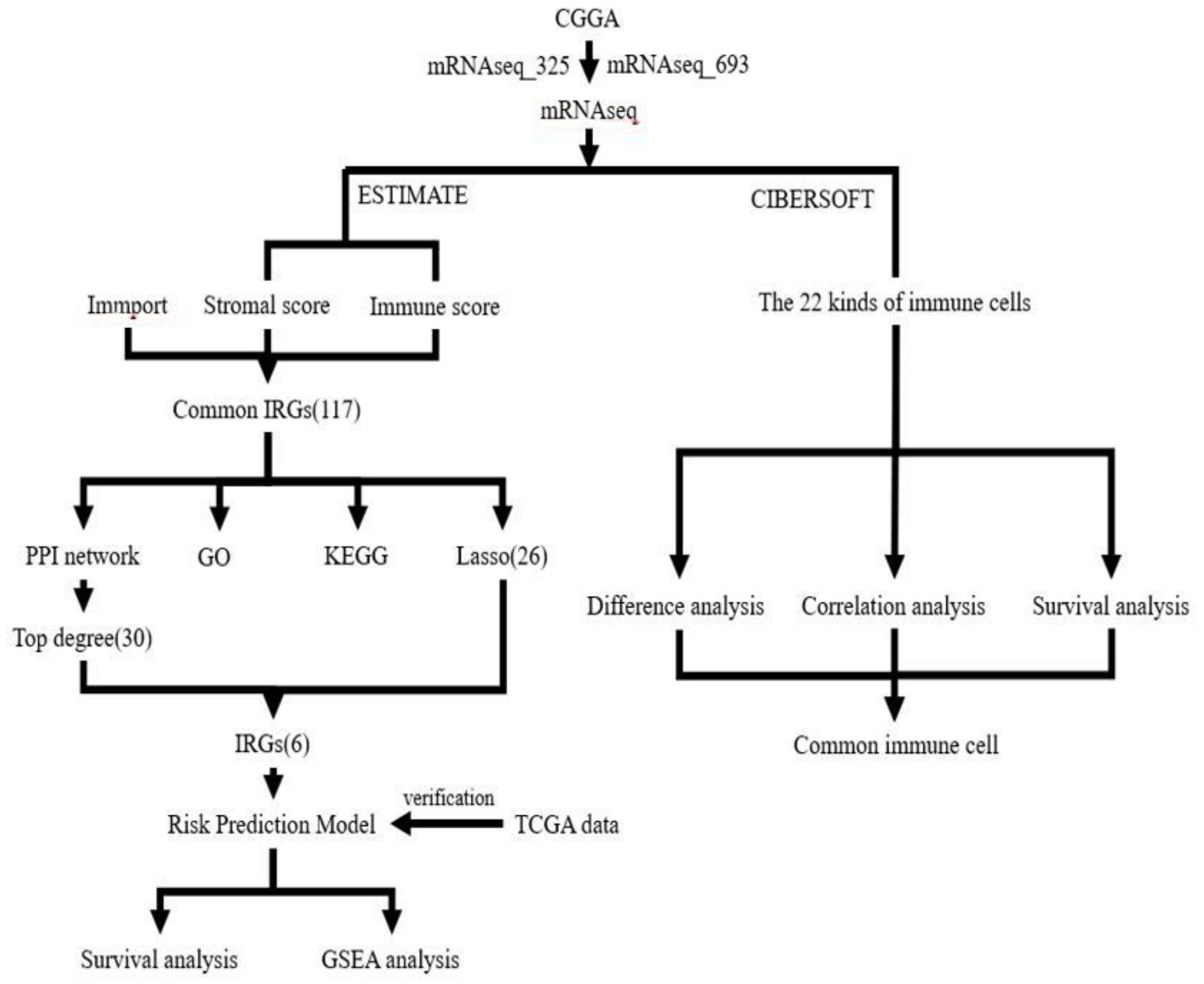

\section{Figure 1}

The workflow map of the research. The number in the brackets represent the number of genes. CGGA: Chinese Glioma Genome Atlas; TCGA: The Cancer Genome Atlas; IRGs: immune related genes; GSEA: Gene set enrichment analyses; GO: Gene ontology; KEGG: Kyoto Encyclopedia of Genes and Genomes. 


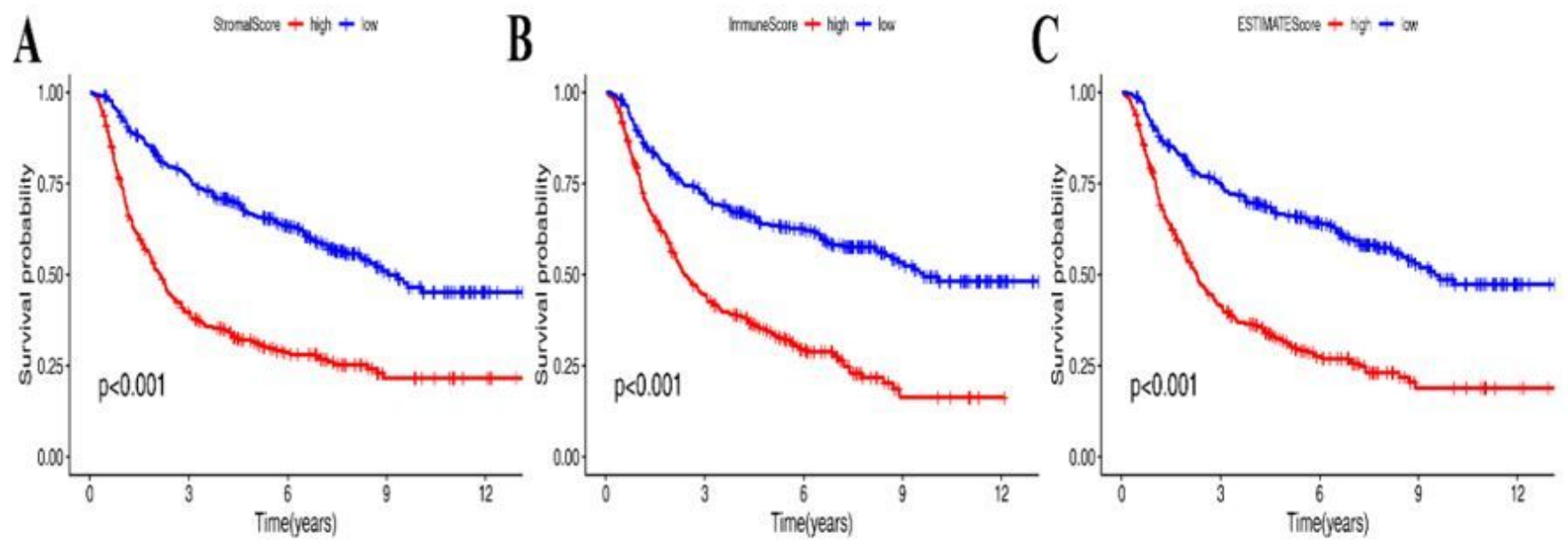

Figure 2

Survival analysis results of Stromal score, Immune score and Estimate score of glioma samples(A\&B\&C). $\mathrm{X}$-axis represents survival rate, $\mathrm{Y}$-axis represents survival time. 


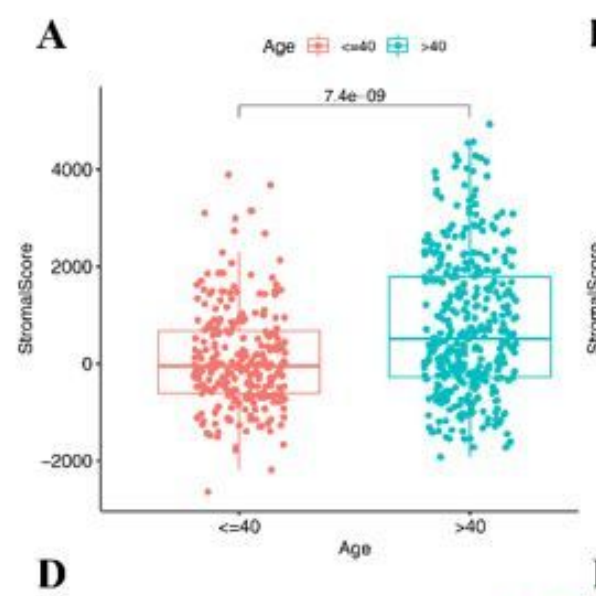

Chemo_status (TMZ treated=1; un-treated=0) क्ष 0 直
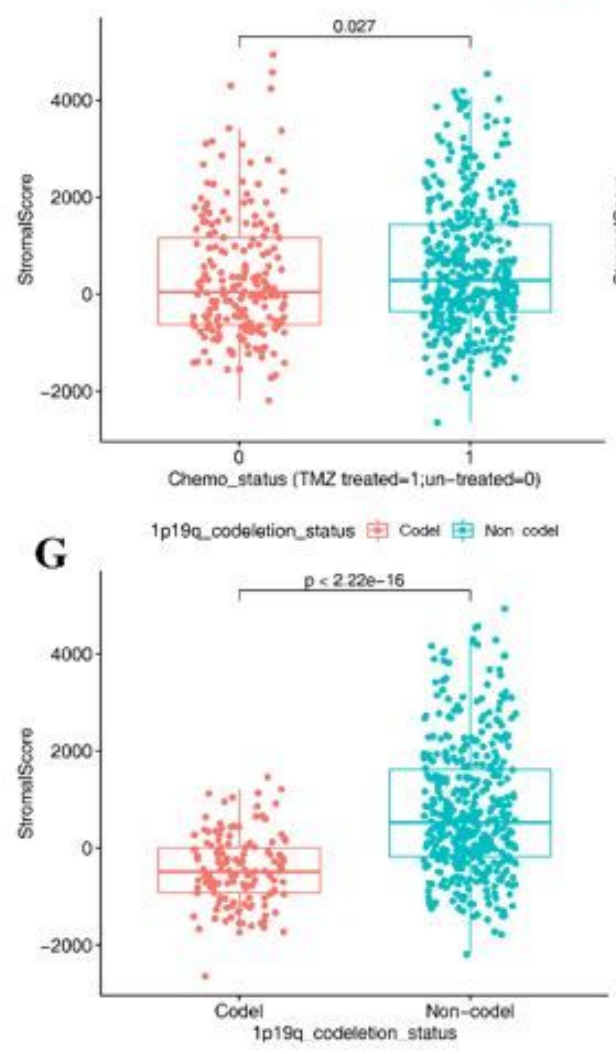

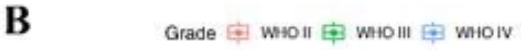

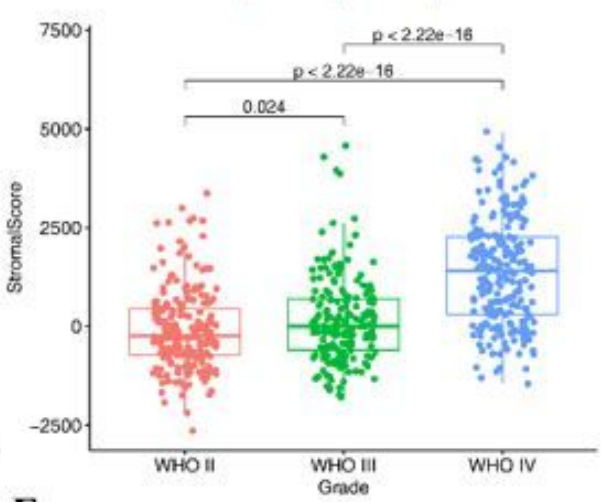

E

MGMTp_methylation_status के metylated it un-metylated

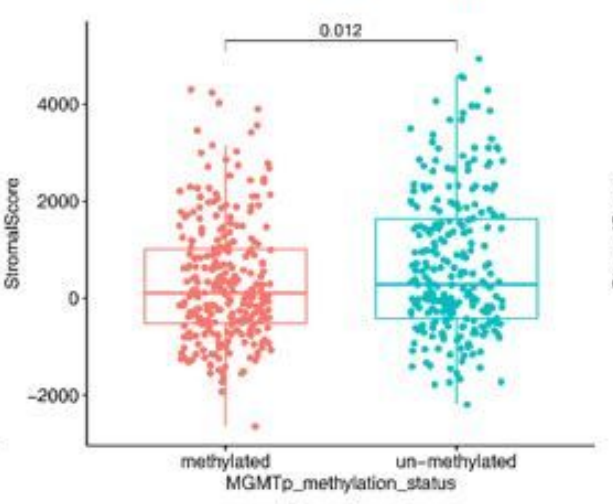

C Radio_status (treated=1,un-treated=0) के 0 由 1

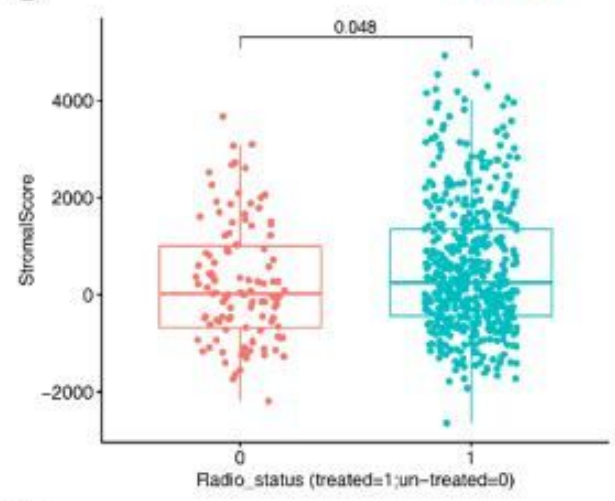

F

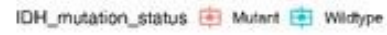

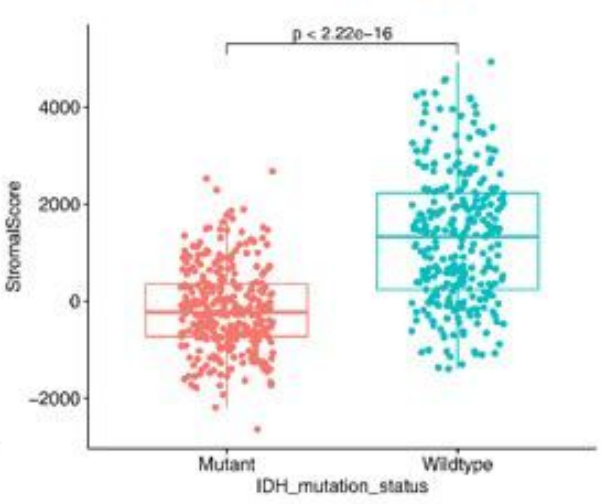

\section{Figure 3}

Relationship between Stromal scores and GBM clinical characteristics. (A-G) Distributions of Stromal scores among different GBM Age, Grade, Radio status, Chemo status, MGMTp methylation status, IDH mutation status and $1 p 19 q$ codeletion status. The $X$-axis represents the stromal score, the $Y$-axis represents the different categories, the value on the graph represents the $P$ value, and $P<0.05$ represents statistical significance. TMZ: Temozolomide; MGMT: Methylation of O6-methylguanine-DNA methyltransferase; IDH: Isocitrate Dehydrogenase. 
A
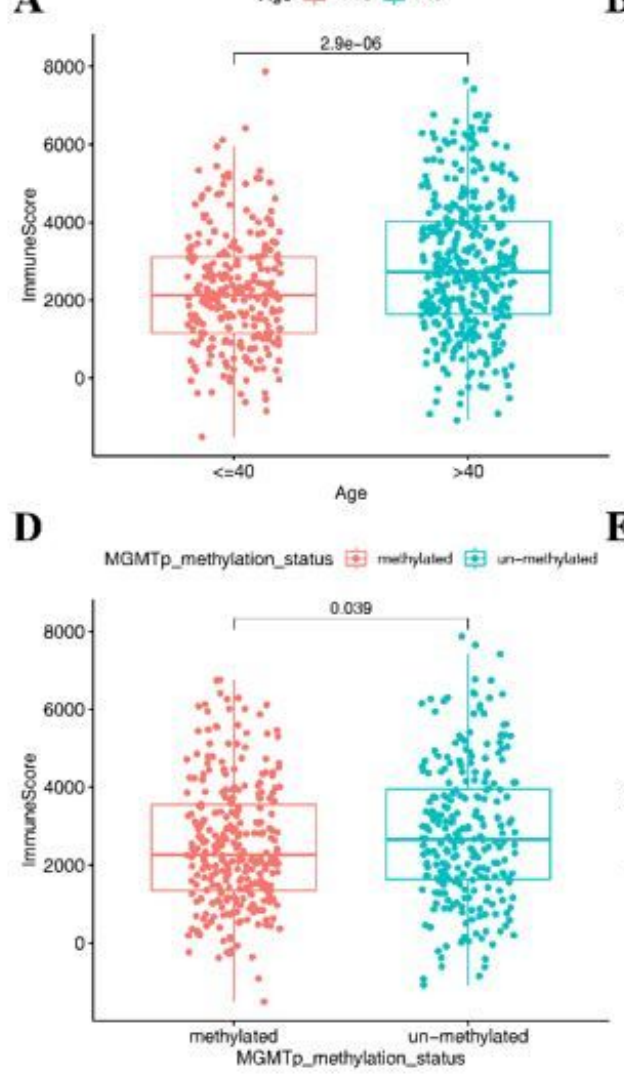

B

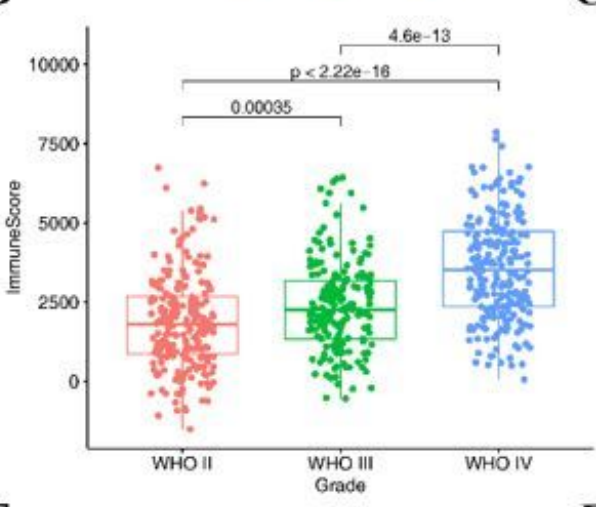

$\mathbf{E}$

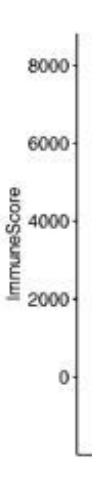

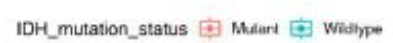

$\mathbf{F}$
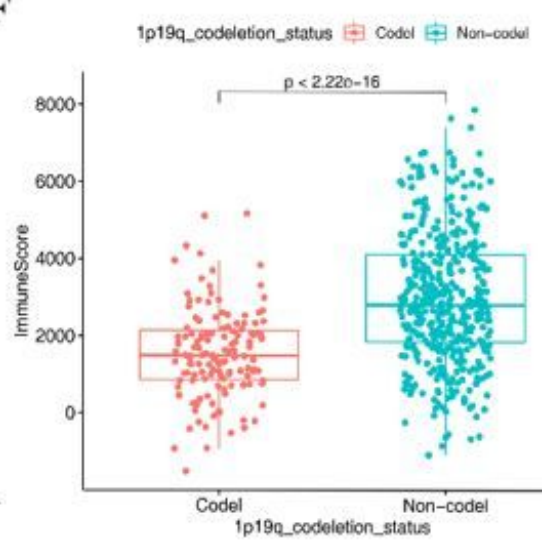

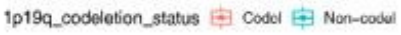

$p<2220-16$
Rado_status (treated=1; un-treatedmo) 후 0 由
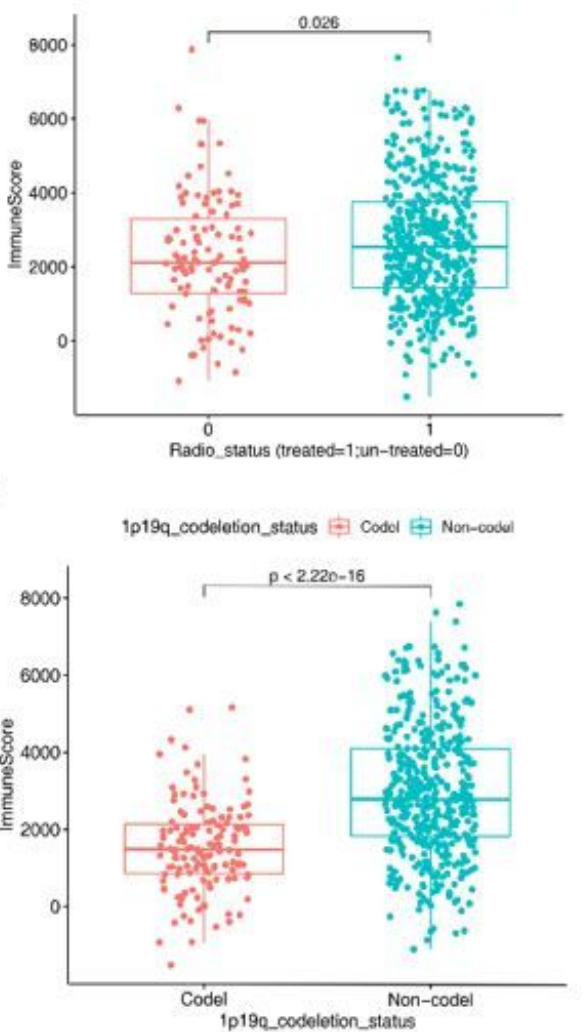

Figure 4

Relationship between immune scores and GBM clinical characteristics. (A-F) Distributions of Stromal scores among different GBM Age, Grade, Radio status, MGMTp methylation status, IDH mutation status and 1 p19q codeletion status. The $X$-axis represents the immune score, the $Y$-axis represents the different categories, the value on the graph represents the $P$ value, and $P<0.05$ represents statistical significance. TMZ: Temozolomide; MGMT: Methylation of 06-methylguanine-DNA methyltransferase; IDH: Isocitrate Dehydrogenase. 

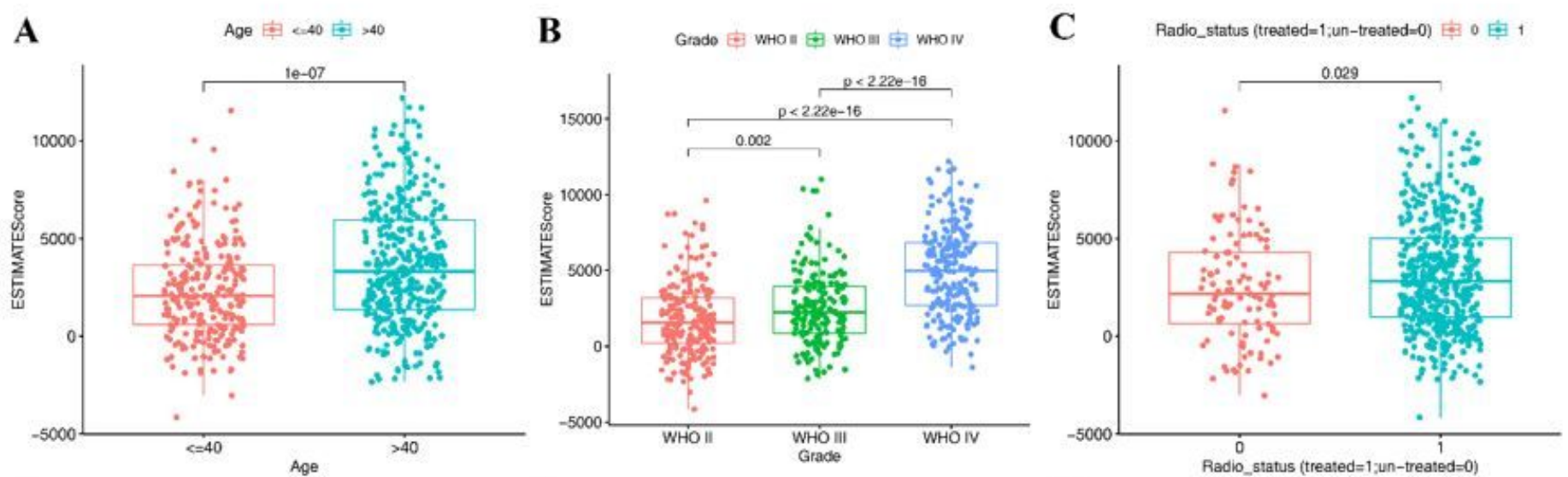

D Chemo status (TMZ treated $=1$; Un-treated 0 ) 审 0 审 ,
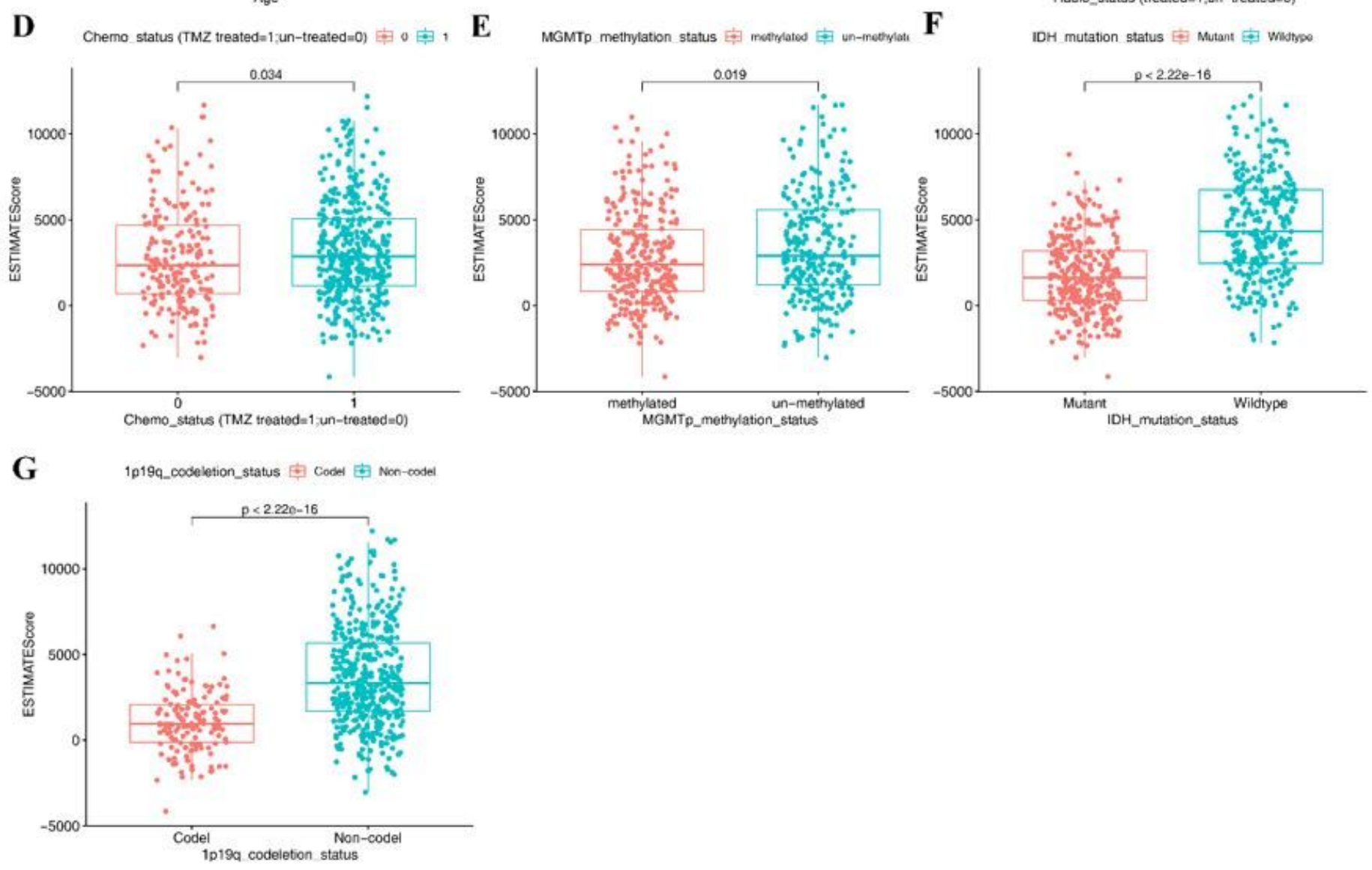

Figure 5

Relationship between estimate scores and GBM clinical characteristics.(A-G) Distributions of ESTIMATE scores among different GBM Age, Grade, Radio status, Chemo status, MGMTp methylation status, IDH mutation status and $1 \mathrm{p} 19 \mathrm{q}$ codeletion status. The $\mathrm{X}$-axis represents the ESTIMATE score, the $\mathrm{Y}$-axis represents the different categories, the value on the graph represents the $P$ value, and $P<0.05$ represents statistical significance. TMZ: Temozolomide; MGMT: Methylation of O6-methylguanine-DNA methyltransferase; IDH: Isocitrate Dehydrogenase. 


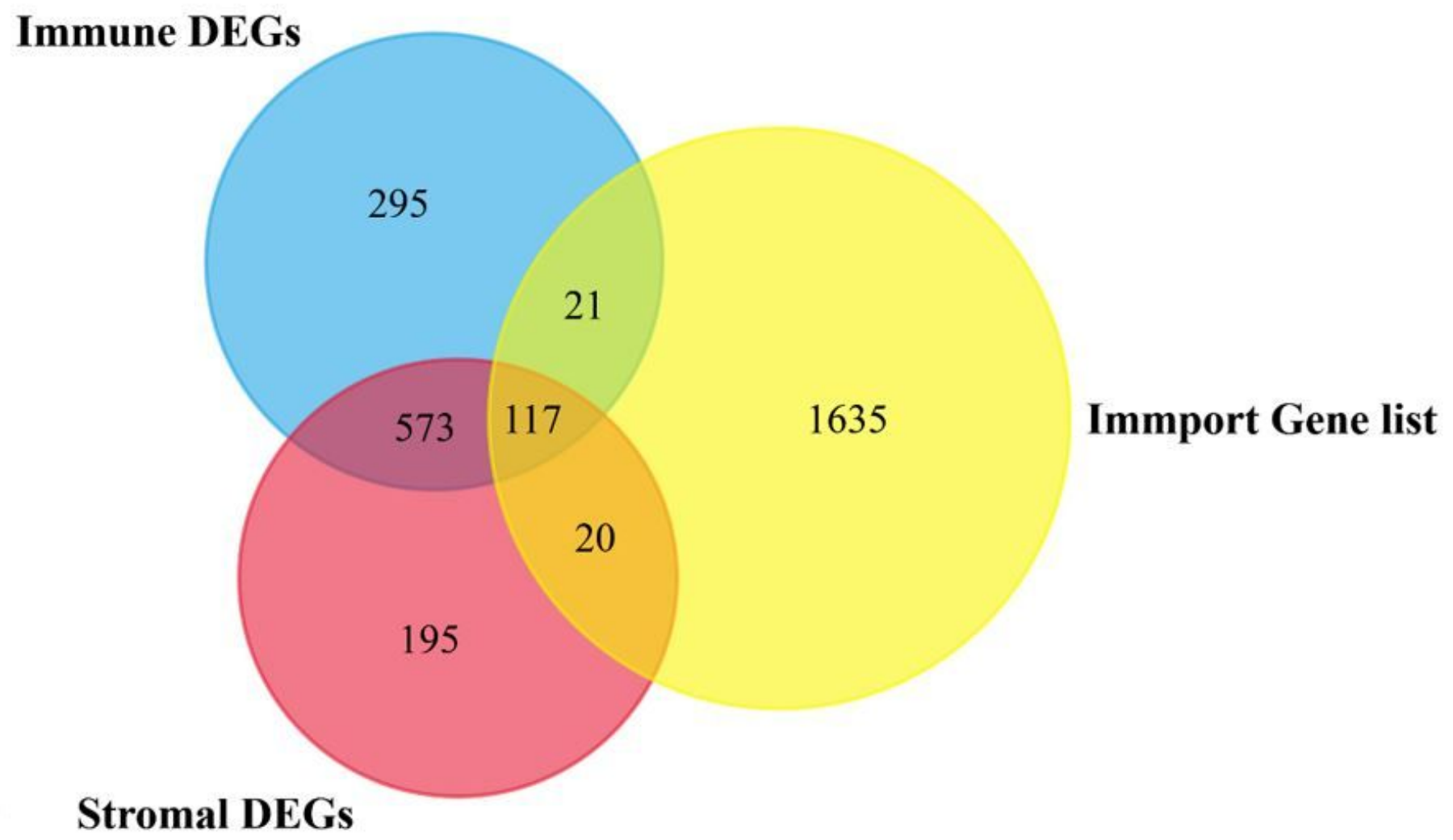

Figure 6

The common immune-related genes of the intersected sets from Stromal score, Immune score and Immport database. 


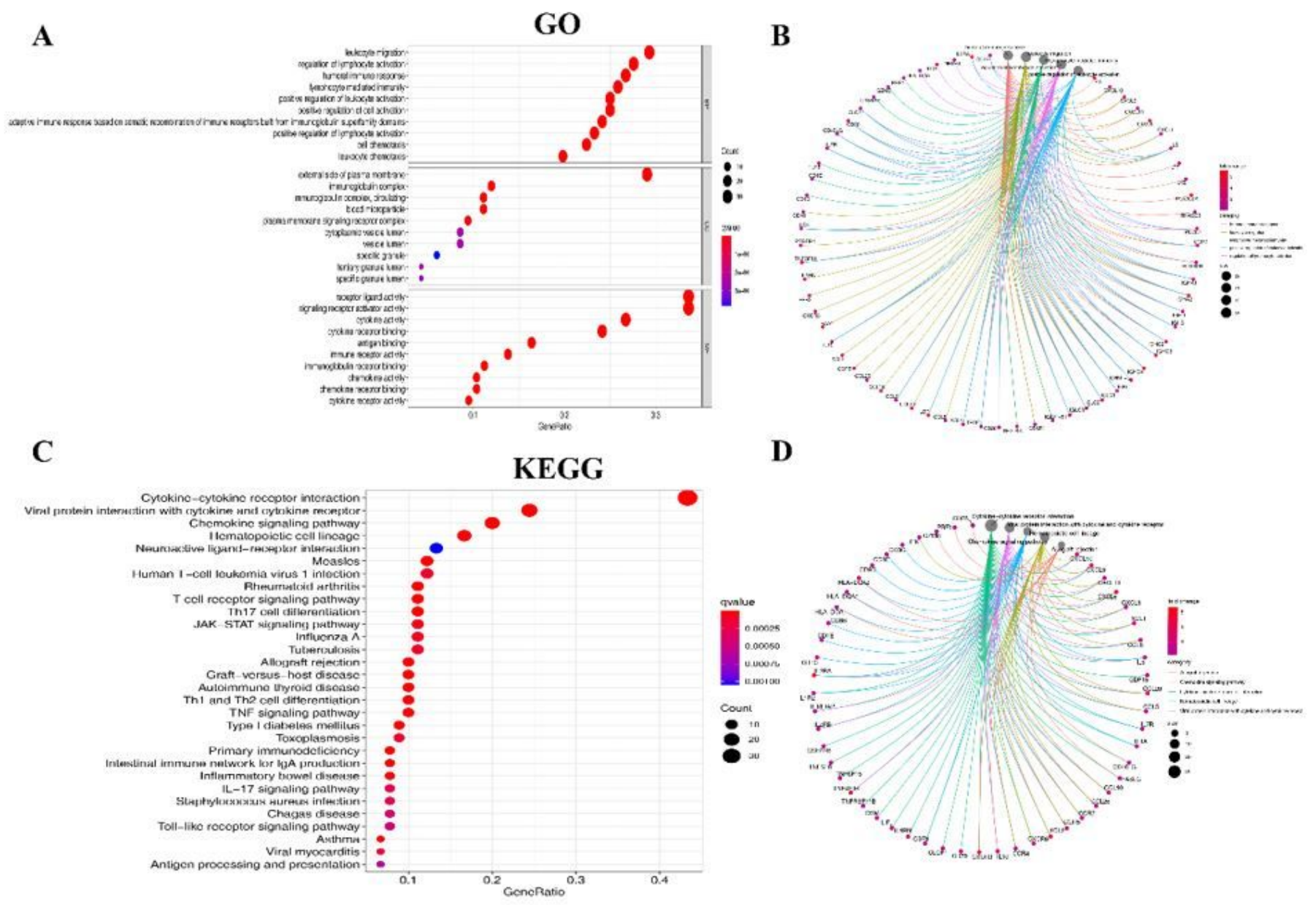

Figure 7

Common immune-related gene enrichment analysis results. (A) Bubble chart results of Gene ontology (GO) terms analysis. (B) Circle diagram of GO terms analysis. (C) Bubble chart results of Kyoto Encyclopedia of Genes and Genomes (KEGG) pathway analysis. (D) Circle diagram of KEGG pathway analysis. BP: biological process; CC: cellular component; MF: molecular function. 

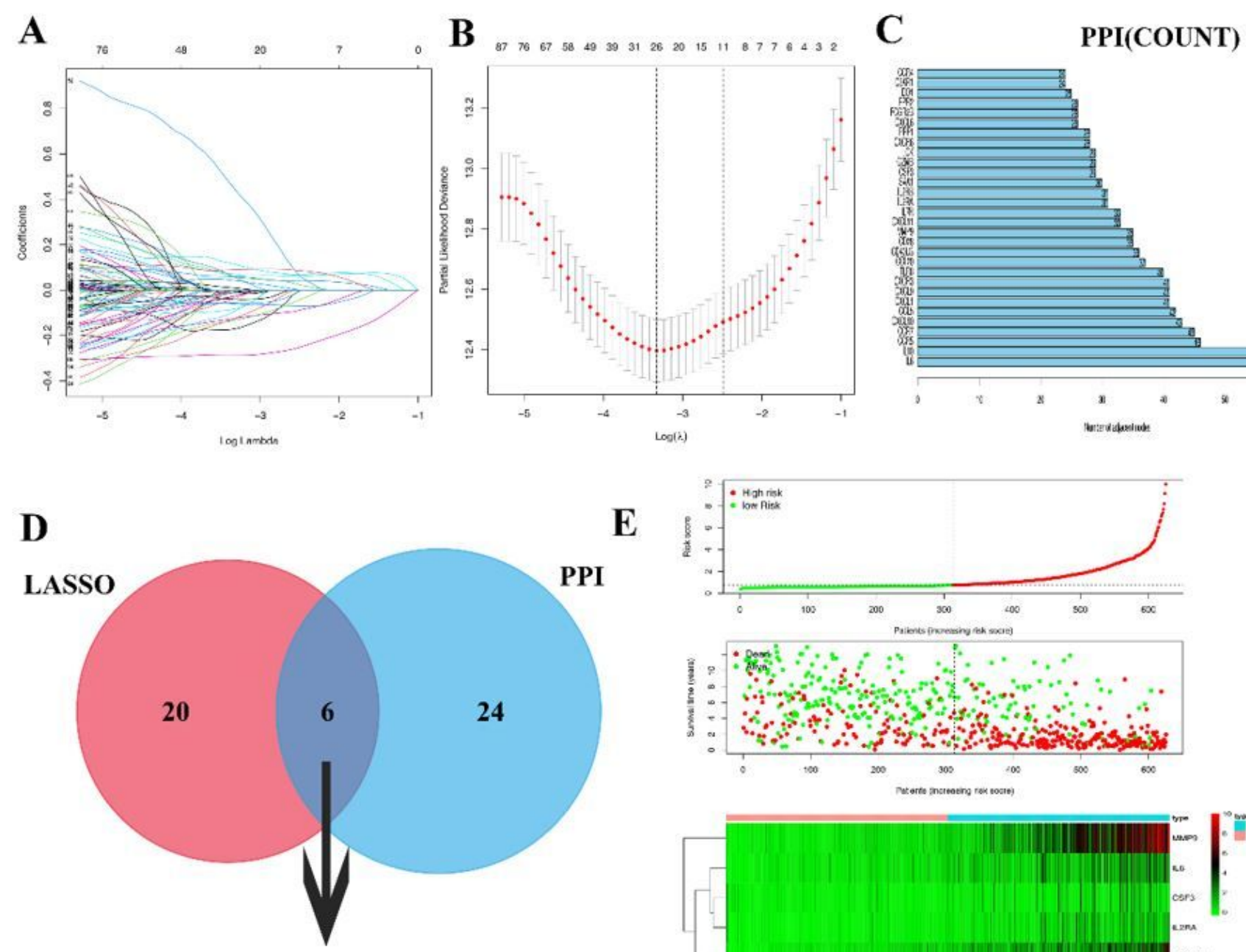

CXCL10, IL6, MMP9, CCL5, CSF3, IL2RA
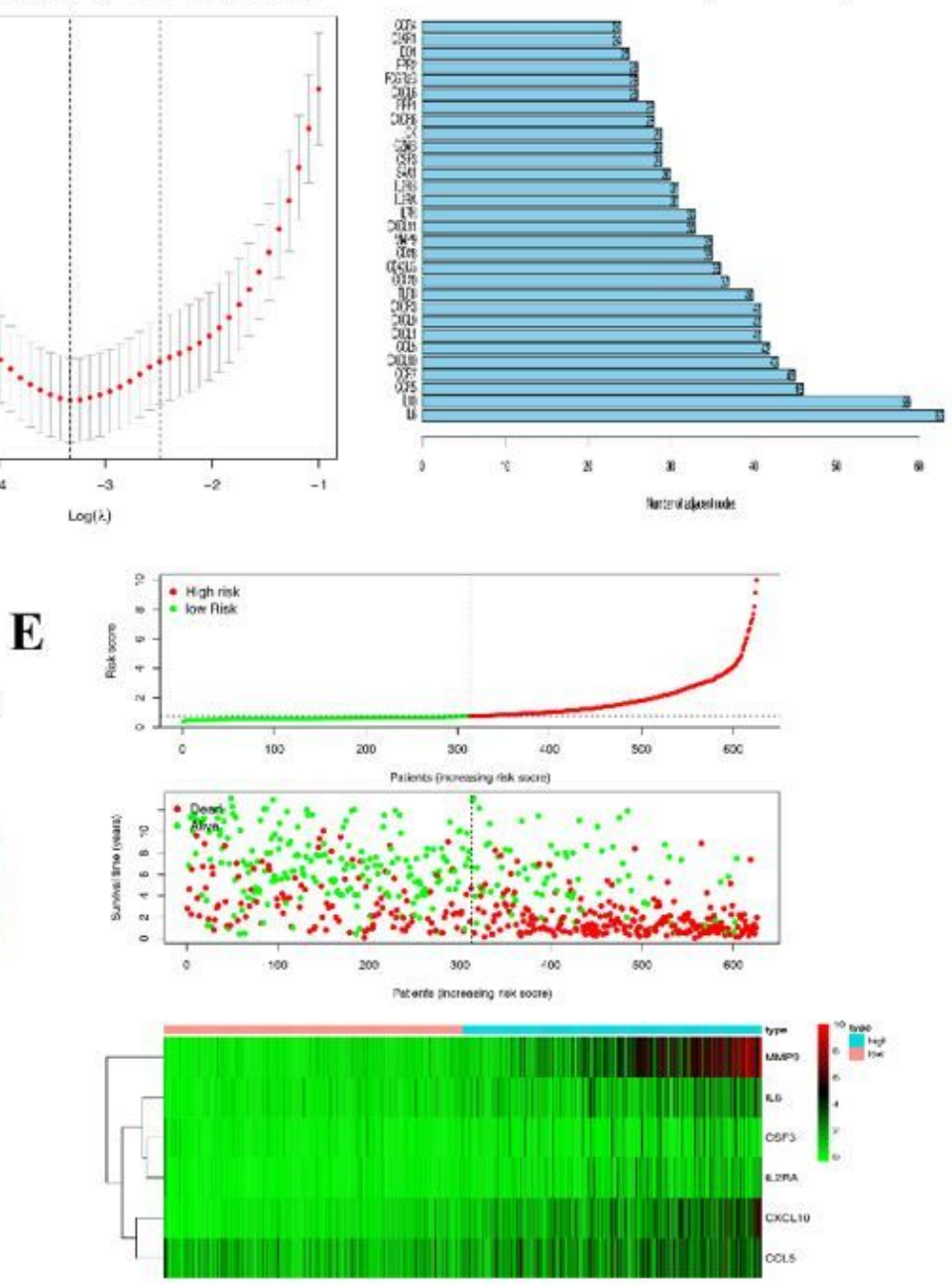

\section{Figure 8}

Identification of target genes and establishment of risk prediction models. (A\&B) 117 IRGs were screened through univariate Cox filtering and Lasso regression analysis to obtain genes related to prognosis. (C) The top 30 high-connection genes in the PPI network. (D) PPI network gene and Lasso regression analysis take the intersection to screen target genes. (E) A risk prediction model constructed using six target genes. In the risk score map, green represents the low-risk group, and red represents the high-risk group. In the survival time map, green represents survival and red represents death. In the heat map, blue represents the high-risk group, pink represents the low-risk group, in the heat map red represents high expression, green represents low expression, and black represents medium expression. PPI: Protein Protein Interactions. 

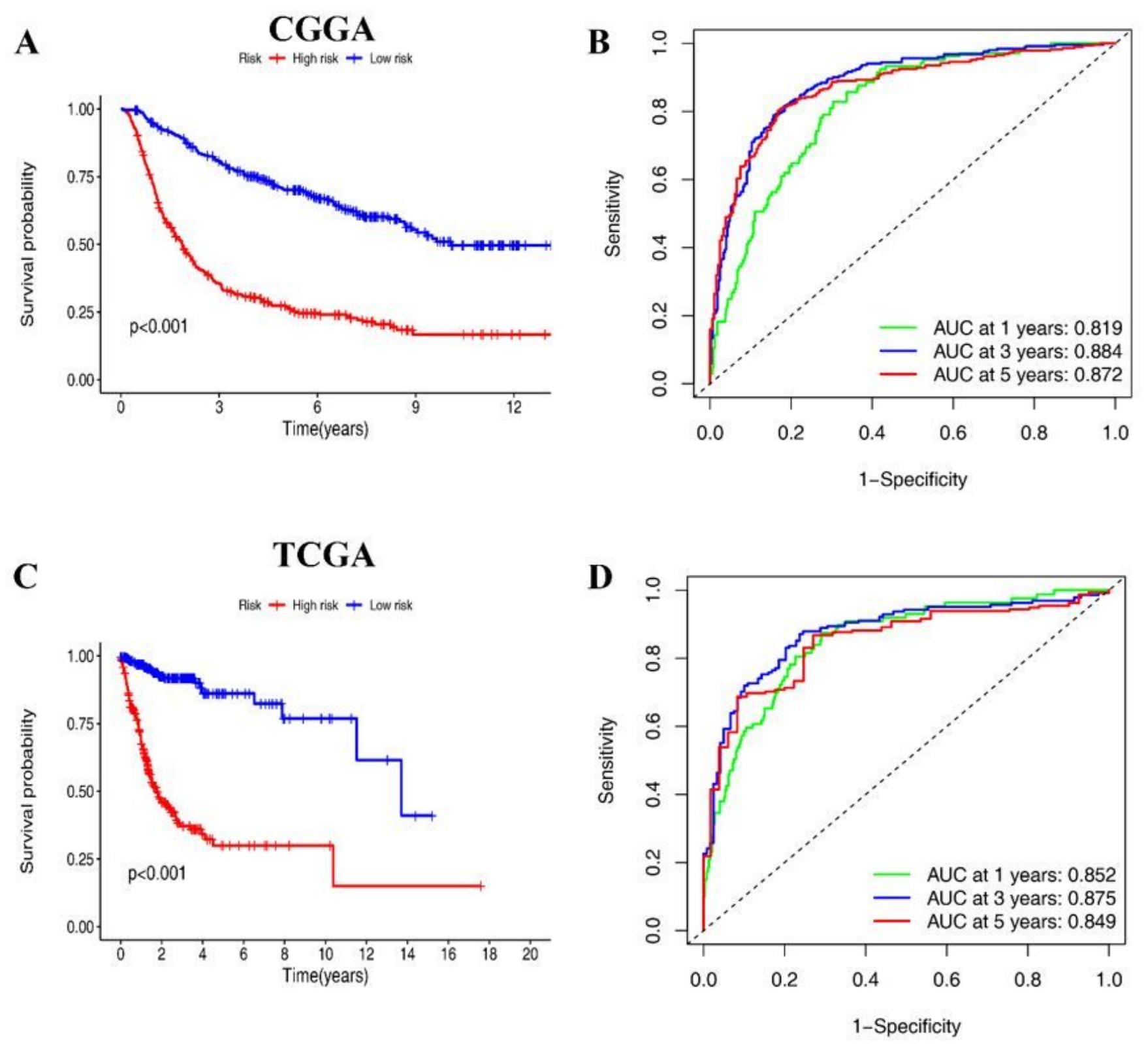

\section{Figure 9}

The results of the survival analysis and model prediction ability of the risk prediction model in CGGA (A) and TCGA (C). The $X$-axis in A\&C represents survival time, the $Y$-axis represents survival rate, the red curve represents the high-risk group, and the blue represents the low-risk group. In $B \& D$, the $X$-axis represents the false positive rate, the $\mathrm{Y}$-axis represents the true positive rate, and the curves of different colors represent different times; CGGA: Chinese Glioma Genome Atlas; TCGA: The Cancer Genome Atlas; AUC: area under the curve; ROC: Receiver operating characteristic. 
A

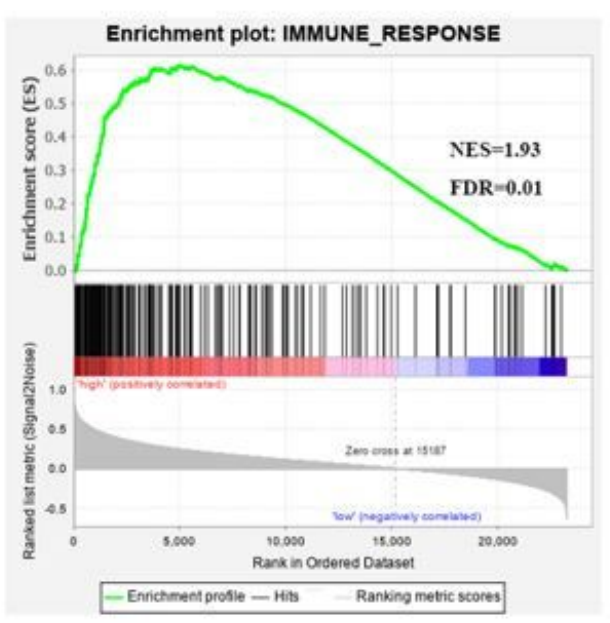

C

Hallmark
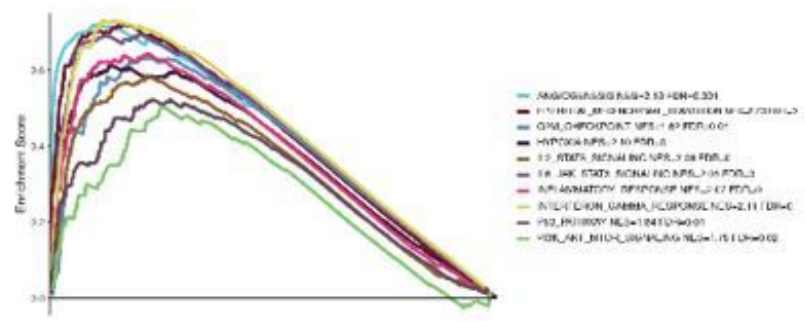

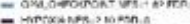

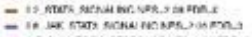

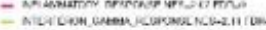

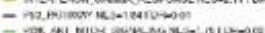

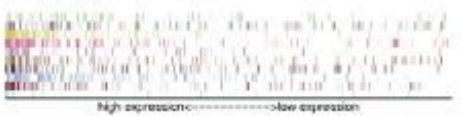

B

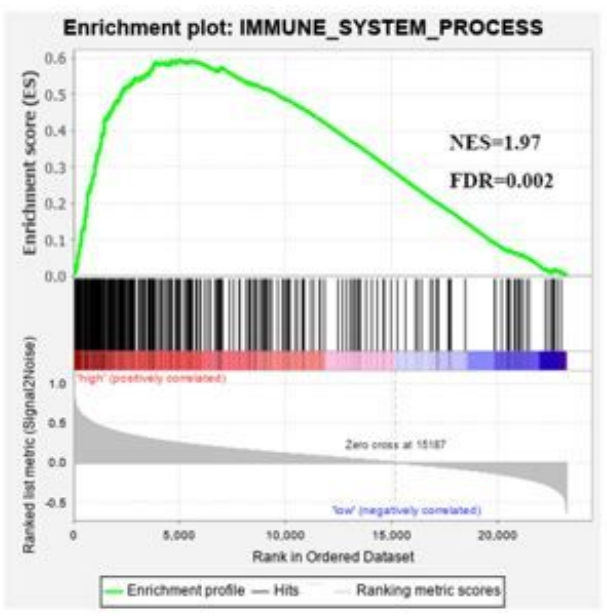

D

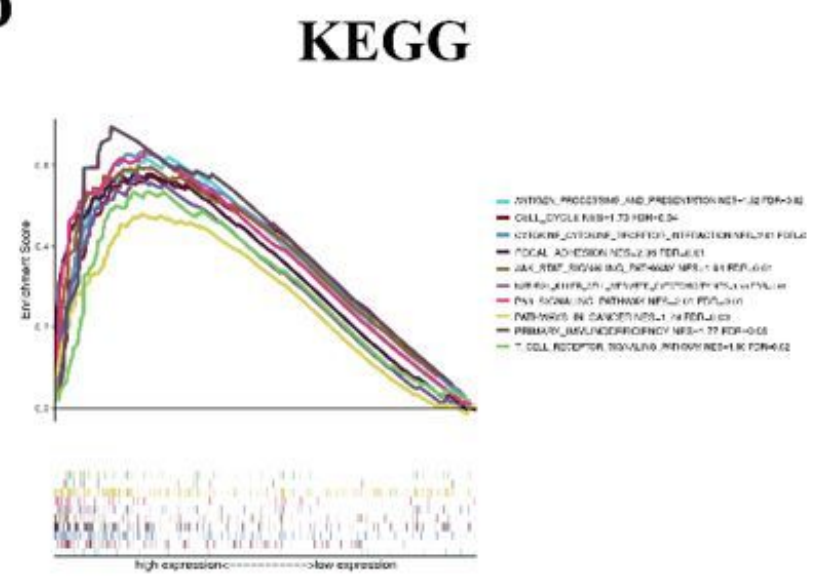

Figure 10

Immune related genes in Immune Response (A), Immune System Process (B) pathway, Hallmark (C), KEGG (D) enrichment analysis results. Different colored curves in C\&D represent different pathways. 

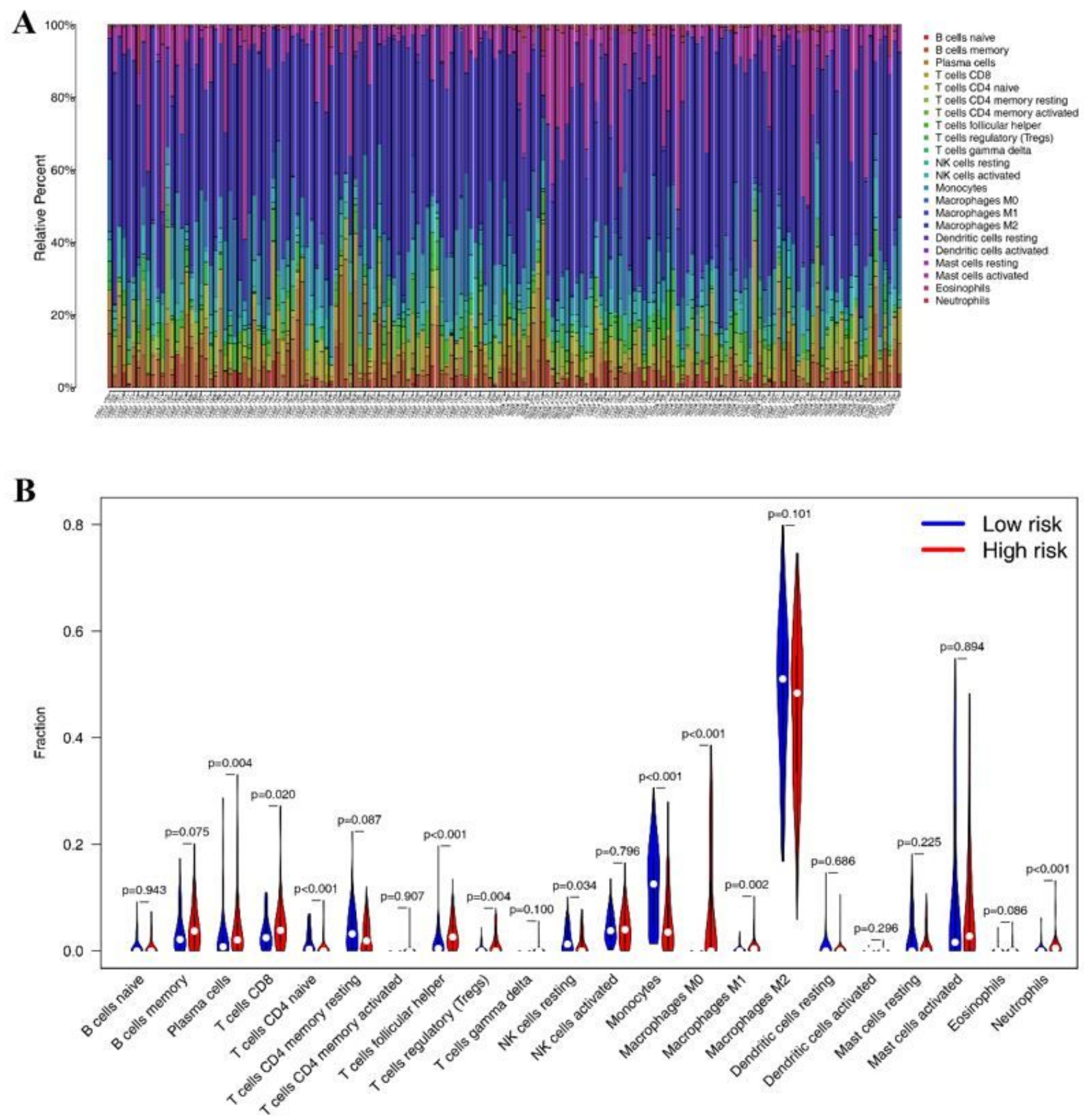

\section{Figure 11}

The content of 22 kinds of immune cells in the glioma sample (A) and the difference analysis results of the immune cells in the high and low risk groups (B) in the risk prediction model. The X-axis in A represents different samples, and the $Y$-axis represents the content of different immune cells, where different colors represent different cells. The X-axis in B represents different immune cells, and the $\mathrm{Y}$-axis contains different immune cells. Red represents the high-risk group and blue represents the low-risk group. 


\section{DE_correlation}

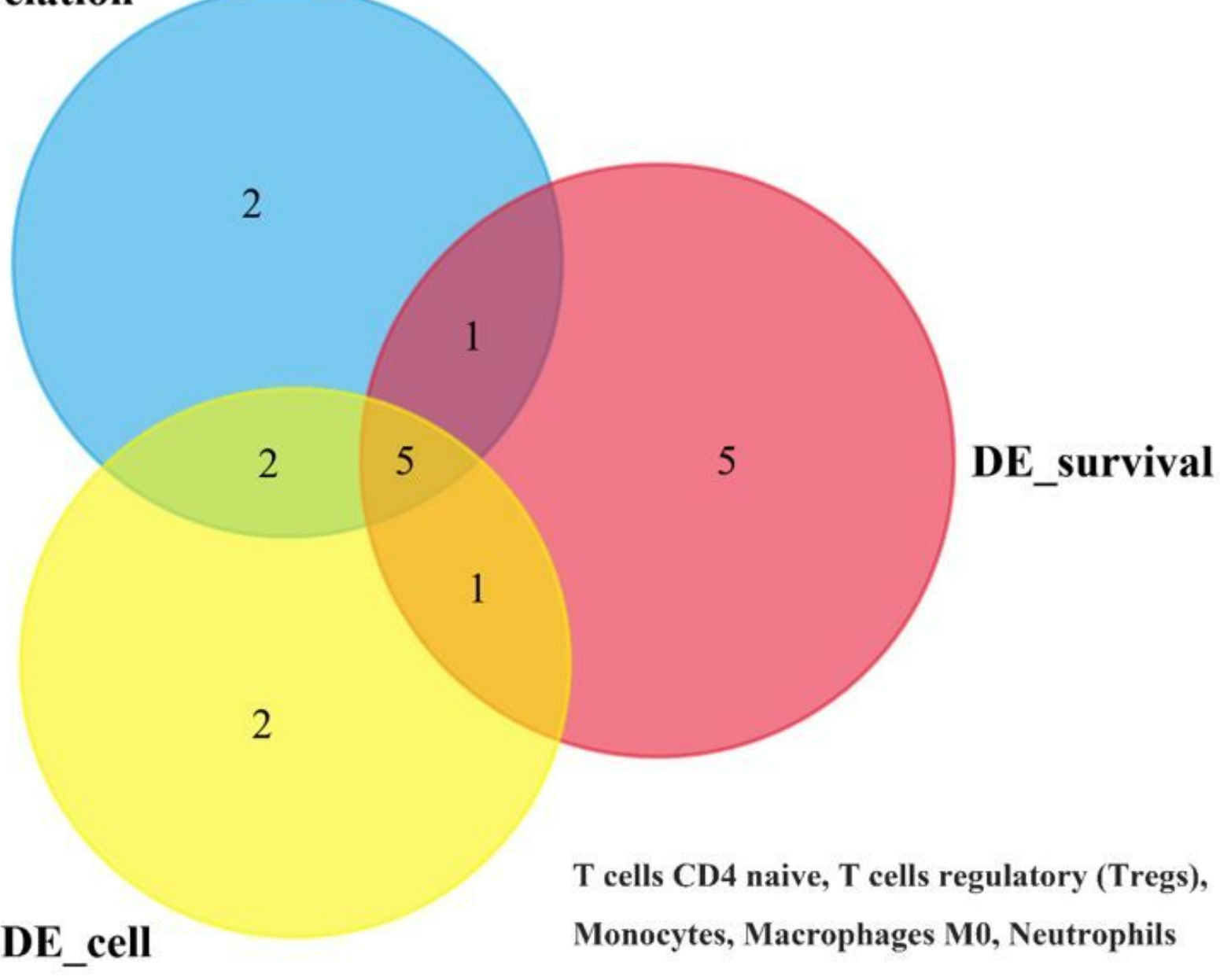

Figure 12

Screening of the common immune cells in the microenvironment of glioma by the Venn diagram for differential expression of immune cells (DE_cell), immune cell correlation (DE_correlation) and immune cell survival analysis (DE_survival). 

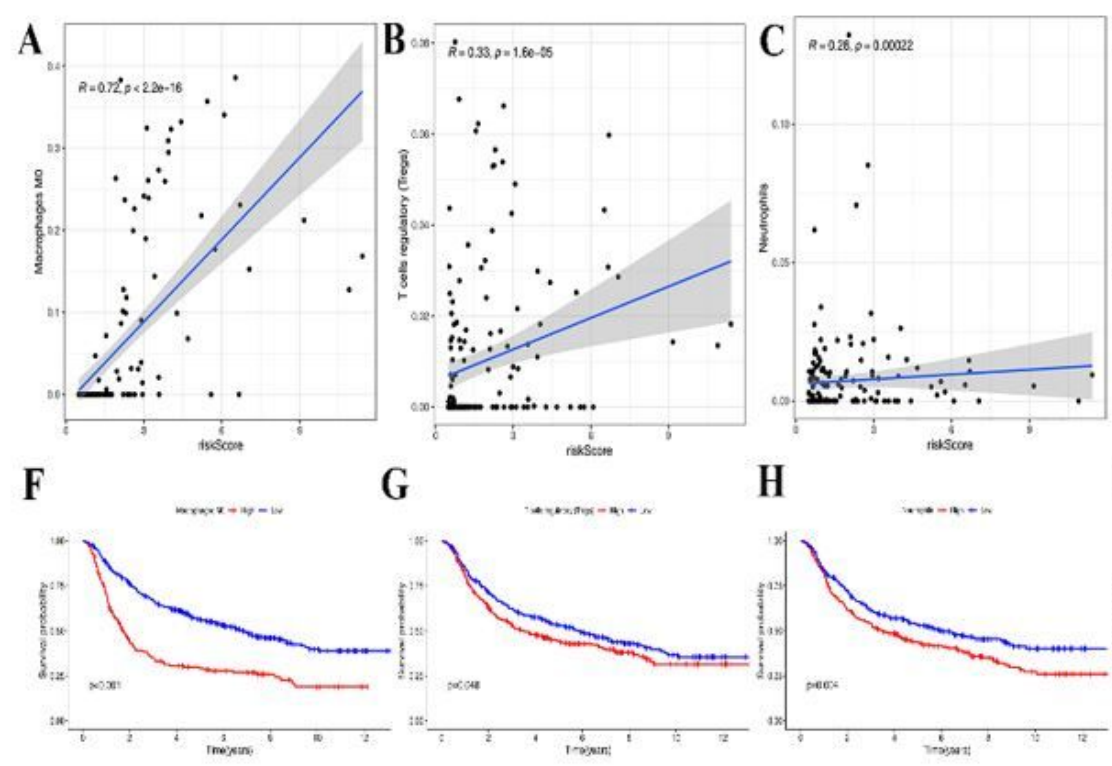

G

$\mathrm{H}$
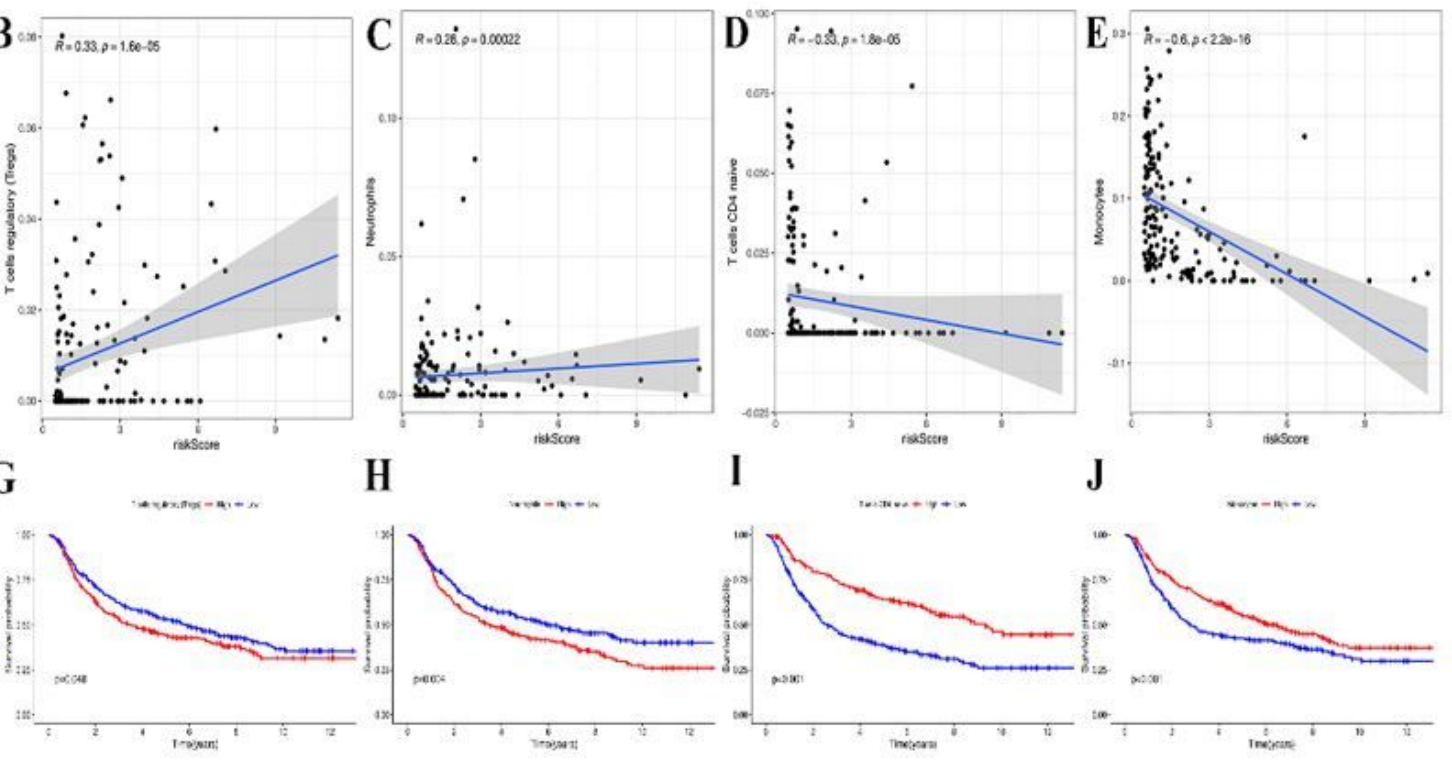

\section{Figure 13}

Correlation analysis and survival analysis results of common immune cells. In (A\&B\&C\&D\&E), the X-axis represents the risk score, the $\mathrm{Y}$-axis represents the content of immune cells, where $\mathrm{R}$ represents correlation, and $P$ value $<0.05$ is statistically significant. (F\&G\&H\&I\&J) The $X$-axis represents the survival time, the $\mathrm{Y}$-axis represents the survival rate, the red curve represents the high-risk group, and the blue represents the low-risk group. 